\title{
Amendment of the Japanese Consensus Guidelines \\ for Autoimmune Pancreatitis, 2013 \\ II. Extrapancreatic lesions, differential diagnosis
}

\author{
Shigeyuki Kawa $\cdot$ Kazuichi Okazaki $\cdot$ Terumi Kamisawa $\cdot$ Keishi Kubo $\cdot$ Hirotaka Ohara Osamu Hasebe $\cdot$ \\ Yasunari Fujinaga $\cdot$ Atsushi Irisawa $\cdot$ Kenji Notohara $\cdot$ Tetsuhide Ito $\cdot$ Kazuo Inui $\cdot$ Hiroyuki Irie $\cdot$ \\ Takayoshi Nishino - Isao Nishimori $\cdot$ Shigeki Tanaka $\cdot$ Toshimasa Nishiyama $\cdot$ Koichi Suda $\cdot$ Keiko Shiratori \\ Masao Tanaka - Tooru Shimosegawa - The Working Committee of the Japan Pancreas Society and the Research \\ Committee for Intractable Pancreatic Disease supported by the Ministry of Health, Labour and Welfare of Japan
}

Received: 3 February 2014/Accepted: 6 February 2014/Published online: 25 March 2014

(C) Springer Japan 2014

\section{Extrapancreatic lesions, differential diagnosis}

\section{II-1. Extrapancreatic lesions}

CQ-II-1-1. What types of extrapancreatic lesions are complicated with AIP?

- A variety of extrapancreatic lesions are reported to be complicated with AIP. Among those cited, those closely associated with AIP include lachrymal and salivary gland lesions, hilar lymphadenopathy, interstitial lung disease, sclerosing cholangitis, retroperitoneal fibrosis, and tubulointerstitial nephritis.

This article is the second of a three-article series on the Japanese consensus guidelines. Please see the first article in the series (doi:10. 1007/s00535-014-0942-2) for the abstract and keywords. The members of the Working Committee are listed in "Appendix" in the text.

\section{S. Kawa $(\square)$}

Center for Health, Safety and Environmental Management,

Shinshu University, Matsumoto, Japan

e-mail: skawapc@ shinshu-u.ac.jp

K. Okazaki

Department of Gastroenterology and Hepatology, Kansai

Medical University, Osaka, Japan

\section{T. Kamisawa}

Department of Internal Medicine, Tokyo Metropolitan

Komagome Hospital, Tokyo, Japan

\section{K. Kubo}

Department of Internal Medicine, Shinshu University School of

Medicine, Matsumoto, Japan
Description A variety of extrapancreatic lesions are reported to be complicated with AIP. Those most closely associated with AIP include lachrymal and salivary gland lesions (Fig. 1) [1], hilar lymphadenopathy [2], interstitial lung disease [3, 4], sclerosing cholangitis $[5,6]$, retroperitoneal fibrosis (Fig. 2) [7], and tubulointerstitial nephritis $[8,9]$. AIP has also been reported to be associated with hypophysitis [10], pachymeningitis [11], autoimmune neurosensory hearing loss [12], uveitis [13], chronic thyroiditis [14], pseudotumors (breast, lung, liver) [15-17], gastric ulcer [18], swelling of the papilla of Vater [19], IgG4 hepatopathy [20, 21], aortitis [22], prostatitis [23], IgG4-related perineural disease [24], Schönlein-Henoch purpura [12], and autoimmune thrombocytopenia [25]. A few cases have reported other extrapancreatic involvement $[12,26,27]$. While it is not certain that all of these lesions were related to AIP, extrapancreatic lesions related to AIP are prevalent in systemic organs (Table 1) [10, 12, 14, 23,

\section{H. Ohara}

Department of Community-Based Medical Education, Nagoya

City University Graduate School of Medical Sciences, Nagoya, Japan

\section{O. Hasebe}

Department of Gastroenterology, Nagano Municipal Hospital,

Nagano, Japan

Y. Fujinaga

Department of Radiology, Shinshu University School of Medicine, Matsumoto, Japan

\section{A. Irisawa}

Department of Gastroenterology, Fukushima Medical University Aizu Medical Center, Aizuwakamatsu, Japan 


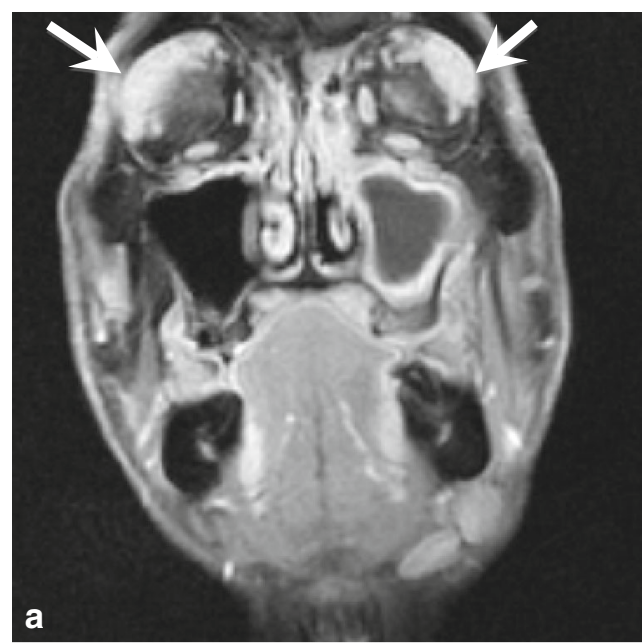

Fig. 1 Swelling of lachrymal and salivary glands (submandibular glands). a Coronal section of the skull. Enhanced MRI shows bilateral swollen lachrymal glands (arrows). b Coronal section of the skull.

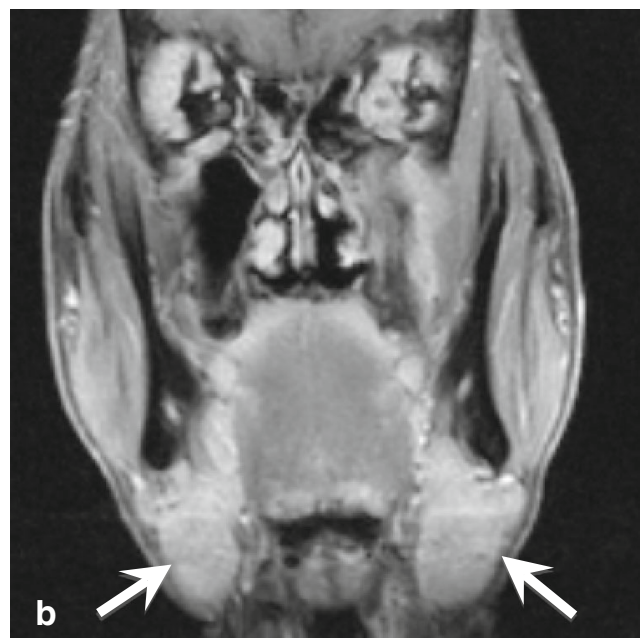

Enhanced MRI (section next to that in panel a) shows bilateral swollen salivary glands (arrows) with homogeneous staining
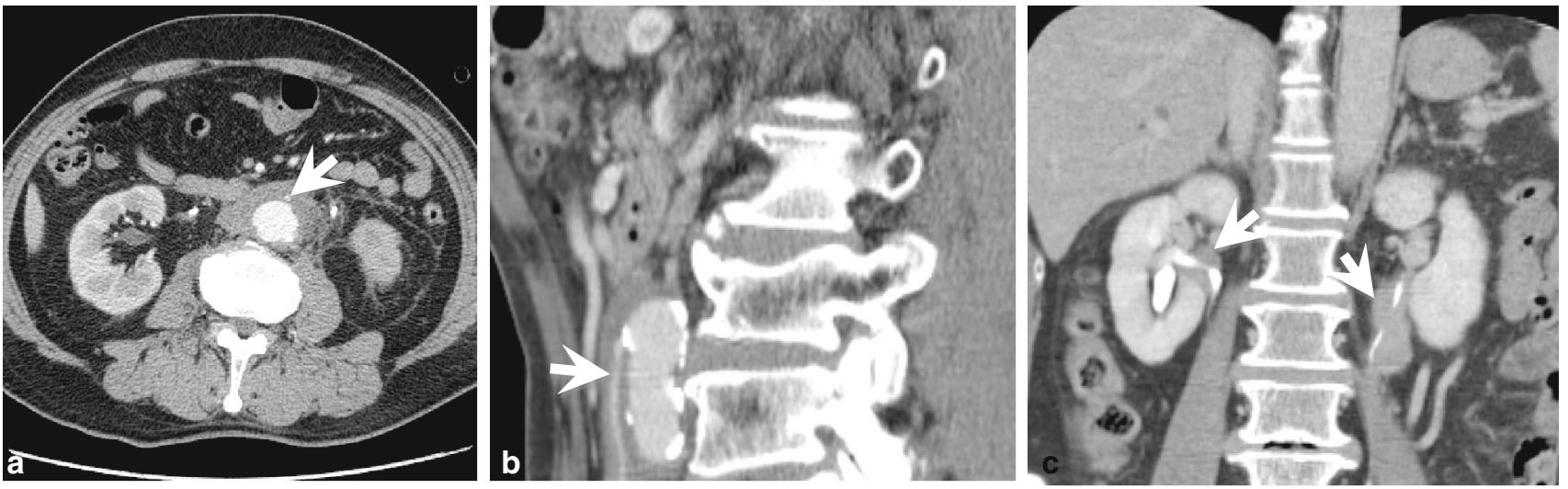

Fig. 2 Retroperitoneal fibrosis. a Enhanced CT (arterial phase) shows an abdominal soft tissue density, indicating a mass around the aorta, which also surrounds the inferior mesenteric artery (arrow).

26-28]. Relative to the pancreatic lesion(s) in AIP, extrapancreatic lesions may appear synchronously or metachronously [29], share the same pathological conditions,

\section{K. Notohara}

Department of Anatomic Pathology, Kurashiki Central Hospital, Kurashiki, Okayama, Japan

\section{T. Ito}

Department of Medicine and Bioregulatory Science, Graduate School of Medical Sciences, Kyushu University, Fukuoka, Japan

\section{K. Inui}

Department of Gastroenterology, Second Teaching Hospital, Fujita Health University, Nagoya, Japan

\section{H. Irie}

Department of Radiology, Faculty of Medicine, Saga University, Saga, Japan and the inferior mesenteric artery (arrow). c CT shows bilateral soft tissue densities, indicating masses around the ureters (arrows)

and show favorable response to corticosteroid therapy. These characteristics indicate a common pathophysiological background, which suggests the presence of a systemic

\section{T. Nishino}

Department of Gastroenterology, Yachiyo Medical Center, Tokyo Women's Medical University, Yachiyo, Japan

I. Nishimori

Nishimori Clinic, Kochi, Japan

\section{S. Tanaka}

Department of Acupuncture and Moxibusion, Tokyo Ariake University of Medical and Health Sciences, Tokyo, Japan

\section{T. Nishiyama}

Department of Public Health and Hygiene, Kansai Medical University, Osaka, Japan b CT shows a soft tissue density indicating a mass around the aorta 
IgG4-related disease [30]. The lesions are typically detected with imaging (CT, MRI, gallium scintigraphy, FDGPET) $[2,31,32]$ and blood tests (hormone assays), but results should be confirmed with histological findings. Extrapancreatic lesions sometimes mimic or are misdiagnosed as primary lesions in the corresponding organ. For example, lachrymal and salivary gland lesions may be mistaken for Sjögren's syndrome, respiratory lesions may be mistaken for sarcoidosis, and sclerosing cholangitis may be mistaken for primary sclerosing cholangitis (PSC). Therefore, it is necessary to distinguish between IgG4related diseases and diseases that arise from the corresponding organ. When a pancreatic lesion is obscure, it may be difficult to detect IgG4-related extrapancreatic lesions. However, recognition of these extrapancreatic lesions should aid in accurate diagnosis of AIP.

\section{CQ-II-1-2. How are extrapancreatic lesions diagnosed?}

- The diagnosis of extrapancreatic lesions complicated with AIP is based on clinical findings that suggest a close association between the lesion and AIP activity, including characteristic pathological findings, a favorable response to corticosteroid therapy, and distinct differentiation from similar lesions due to other causes in the corresponding organ. (Level of recommendation: B)

Description Several lines of evidence support the theory that extrapancreatic lesions are associated with AIP, including (1) frequent or coincident occurrence; (2) pathological findings of severe lymphoplasmacytic infiltration and storiform fibrosis, IgG4-positive plasma cell infiltrations, and obliterative phlebitis; (3) favorable response to corticosteroid therapy or synchronous response to therapies; and (4) distinct differentiation from the lesions of the corresponding organ, such as a distinction between AIPassociated salivary gland lesions and those due to Sjögren's syndrome. Among the many possible extrapancreatic lesions listed in Table 1, the following fulfill the above criteria: lachrymal and salivary gland lesions, respiratory

\section{K. Suda}

Department of Pathology, Tokyo-West Tokushukai Hospital, Tokyo, Japan

\section{K. Shiratori}

Department of Gastroenterology, Tokyo Women's Medical University, Tokyo, Japan

\section{Tanaka}

Department of Surgery and Oncology, Graduate School of Medical Sciences, Kyushu University, Fukuoka, Japan

\section{T. Shimosegawa}

Division of Gastroenterology, Tohoku University Graduate School of Medicine, Sendai, Japan
Table 1 Extrapancreatic lesions that may be complicated with autoimmune pancreatitis

Close association
Lachrymal gland inflammation
Sialadenitis
Hilar lymphadenopathy
Interstitial lung disease
Sclerosing cholangitis
Retroperitoneal fibrosis
Tubulointerstitial nephritis
Possible association
Hypophysitis
Pachymeningitis
Autoimmune neurosensory hearing loss
Uveitis
Chronic thyroiditis
Pseudotumor (breast, lung, liver)
Gastric ulcer
Swelling of papilla of Vater
IgG4 hepatopathy
Aortitis
Prostatitis
IgG4-related perineural disease
Schönlein-Henoch purpura
Autoimmune thrombocytopenia

lesions, sclerosing cholangitis, retroperitoneal fibrosis, and tubulointerstitial nephritis.

CQ-II-1-3.: What are the differences between lachrymal and salivary gland lesions associated with AIP and those associated with Sjögren's syndrome?

- Compared to those of Sjögren's syndrome, AIP-associated lachrymal and salivary gland lesions show normal or slightly impaired exocrine function, presenting as a slight or negligible dryness in the eyes and mouth. (Level of recommendation: B)

- Salivary gland lesions associated with AIP appear predominantly in the submandibular gland, and those associated with Sjögren's syndrome frequently appear in the parotid gland. (Level of recommendation: B)

- Compared with those of Sjögren's syndrome, AIPassociated lachrymal and salivary gland lesions show negative results in tests for SS-A/Ro and SS-B/La autoantibodies. (Level of recommendation: B)

- Compared with those of Sjögren's syndrome, AIPassociated lachrymal and salivary gland lesions show numerous IgG4-positive plasma cell infiltrations in the affected tissues. (Level of recommendation: B)

- Unlike those of Sjögren's syndrome, AIP-associated lachrymal and salivary gland lesions respond favorably to corticosteroid therapy. (Level of recommendation: B) 
Description The presence of symmetrical lachrymal and salivary gland lesions has been noted in approximately 14-39\% of patients with AIP (Fig. 1) [12, 26, 27, 33]. These lesions, which were previously thought to be a complication of Sjögren's syndrome, are now thought to be related to Mikulicz's disease or Küttner's tumor (chronic sclerosing sialadenitis) [34, 35]. Useful findings for differentiating among these possibilities include the following: (1) compared to those of Sjögren's syndrome, AIPassociated lachrymal and salivary gland lesions show normal or slightly impaired exocrine function, presenting as a slight or negligible dryness in the eyes and mouth [33, 36]; (2) salivary gland lesions associated with AIP appear predominantly in the submandibular gland [31], and those associated with Sjögren's syndrome frequently appear in the parotid gland [37]; (3) lachrymal and salivary gland lesions associated with AIP show negative results in tests for SS-A/Ro and SS-B/La autoantibodies [35]; (4) lachrymal and salivary gland lesions associated with AIP show numerous $\operatorname{IgG} 4$-positive plasma cell infiltrations in the affected tissues [35]; and (5) lachrymal and salivary gland lesions associated with AIP respond favorably to corticosteroid therapy [35]. Most lesions are bilateral and symmetrically distributed, but a few cases may exhibit unilateral lesions. To ensure accurate diagnosis, a salivary gland biopsy is preferable, but the less invasive lip biopsy can be substituted for examinations of the small salivary gland. AIP complicated with lachrymal and salivary gland lesions represents a highly active state, with higher serum IgG4 concentrations and more severe pancreatic swelling compared to AIP without complications [27, 38]. When diagnosing AIP-associated lachrymal and salivary gland lesions, it is recommended to refer to the diagnostic criteria for IgG4+ Mikulicz's disease [39].

\section{CQ-II-1-4. What kind of respiratory lesions are asso- ciated with AIP?}

- Respiratory lesions associated with AIP include interstitial lung disease, asthma, inflammatory pseudotumor of the lung, edema and swelling of the tracheobronchial mucosa, thickening of the bronchial wall and bronchial vascular bundle, pleural lesions, and hilar or mediastinal lymphadenopathy. The lesions must be differentiated from idiopathic interstitial pneumonia, sarcoidosis, and lung tumor. Similar to AIP-associated pancreatic lesions, the pathology of these AIP-associated lesions includes numerous IgG4-bearing plasma cell infiltrations and a favorable response to corticosteroid therapy. (Level of recommendation: B)

Description Interstitial lung disease complicated with AIP has been noted in approximately $8-13 \%$ of patients $[3,4]$. This condition exhibited high serum KL-6 levels and alveolar IgG4-bearing plasma cell infiltrations $[3,4,16]$. Thoracic CT showed various lung lesions, bronchial wall thickening, nodules, interlobular thickening, infiltration in the middle and lower lung fields (Fig. 3a, b) [28], and honeycombing in the lower lung field [40]. While IgG4related respiratory lesions of interstitial pneumonia, nodular lesions, localized ground glass-opacity (GGO), and pleural lesions sometimes occurred without a pancreatic lesion [16, 41-45], a definitive diagnosis of IgG4-related respiratory lesions was difficult in patients with intrathoracic lesions alone, as IgG4-bearing plasma cells have also been observed in other types of lung lesions [46].

Another respiratory lesion associated with AIP is inflammatory pseudotumor of the lung [47]. Although inflammatory pseudotumors comprise various subtypes, the lesion associated with AIP corresponds to a plasma cell granuloma, which shows lymphoplasmacytic infiltration, fibrosis, obstructive phlebitis, and IgG4-bearing plasma cell infiltration characteristics that are similar to those of a pancreatic lesion in AIP [47]. In addition, obstructive arteritis is sometimes considered a lung lesion. An inflammatory pseudotumor is often suspected to be a lung tumor, which may result in inappropriate resection. However, unlike a lung tumor, the inflammatory pseudotumor responds favorably to corticosteroid therapy (Fig. 3c, d) [28].

Gallium scintigraphy has revealed hilar and mediastinal lymphadenopathy in $67-75 \%$ of patients with AIP. In these cases, bronchoscopy and CT sometimes reveal edema and swelling of the tracheobronchial mucosa as well as thickening of the bronchial wall and bronchial vascular bundle. While these finding are consistent with the characteristics of sarcoidosis (Fig. 3e, f), patients with AIP showed normal serum angiotensin-converting enzyme (ACE) levels [2, 31, 44, 45, 48, 49].

\section{CQ-II-1-5. How is AIP-associated sclerosing cholangitis differentiated from PSC or biliary malignancies?}

- Differentiation between AIP-associated sclerosing cholangitis (IgG4-related sclerosing cholangitis) and PSC or biliary malignancies should be ascertained carefully based on a combination of clinical features, pathological findings, and imaging tests such as cholangiography, ultrasonography, endoscopic ultrasonography (EUS), intraductal ultrasonography (IDUS), CT, and MRI. (Level of recommendation: A)

Description AIP-associated sclerosing cholangitis, also known as IgG4-related sclerosing cholangitis, is characteristically considered a lower (intrapancreatic) bile duct stenosis, although is sometimes distributed widely across the biliary system. It may exhibit restricted stenosis from the hilar to the extrahepatic bile ducts or multiple stenosis 

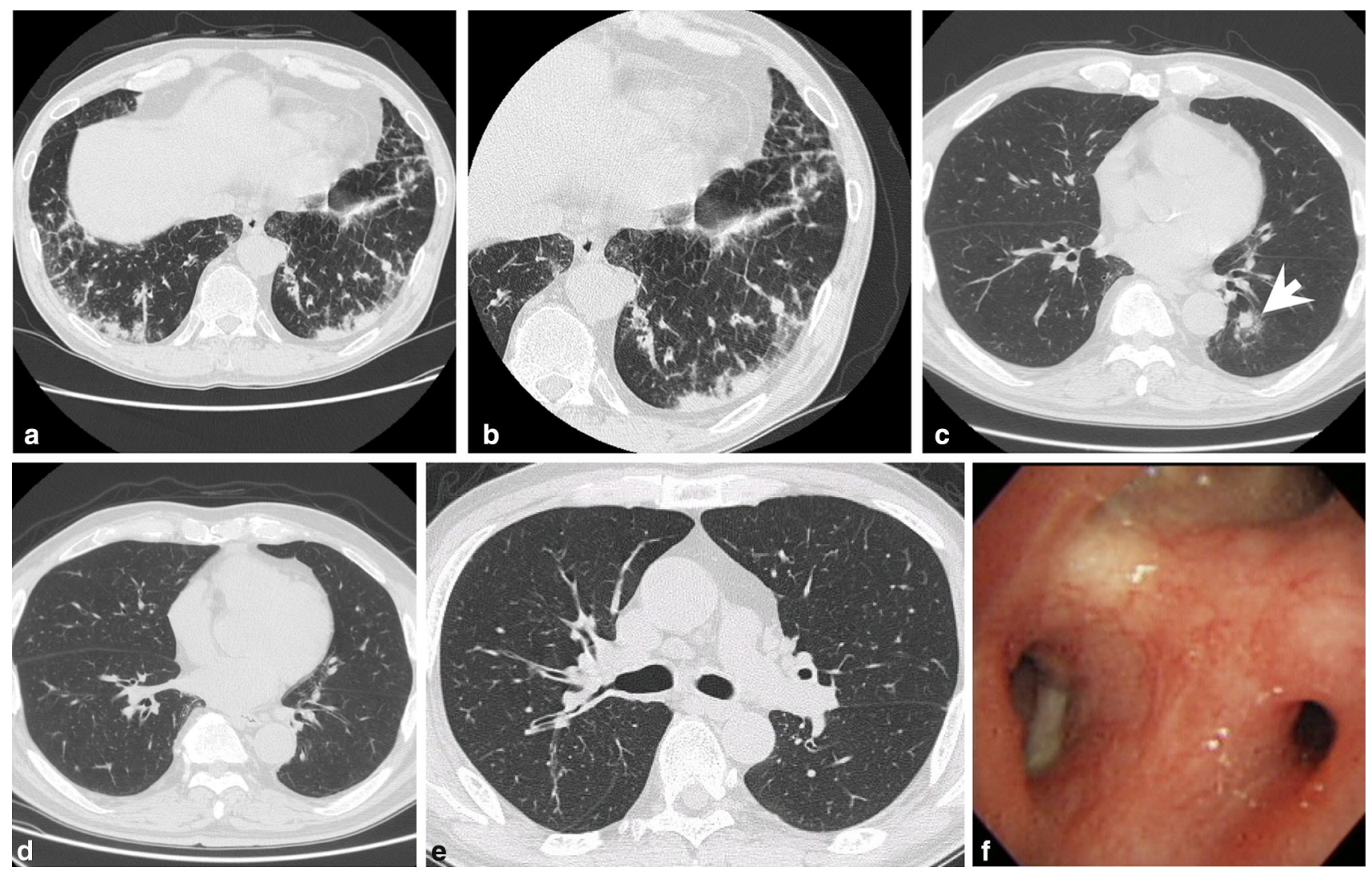

Fig. 3 Various lung lesions associated with AIP. CT shows various lung lesions associated with AIP. a, b Bronchial wall thickening, a nodule, interlobular thickening, and infiltration in the lower lung field. CT shows nodular lesion identified as an inflammatory pseudotumor (arrow), $\mathbf{c}$ before corticosteroid therapy, $\mathbf{d}$ after therapy, the nodular

in the intrahepatic bile ducts (Fig. 4) [50]. The lower bile duct lesions must be differentiated from pancreatic cancer or common bile duct cancer; intrahepatic and hilar bile duct lesions must be differentiated from PSC and cholangiocarcinoma, respectively.

There are several differences between IgG4-related sclerosing cholangitis and PSC. IgG4-related sclerosing cholangitis has shown a preponderance among older males and is frequently complicated with obstructive jaundice [51-53]. In contrast, PSC has been more commonly found in young and middle-aged patients, sometimes complicated with inflammatory bowel diseases [12, 51-53]. Cholangiography of IgG4-related sclerosing cholangitis reveals lower bile duct stenosis and relatively long strictures from the hilar to the intrahepatic biliary systems, with simple distal dilations [51, 52]. Cholangiography of PSC characteristically shows band-like strictures (short strictures of 1-2 mm), a beaded appearance, a pruned tree appearance, or diverticulum-like outpouching (Fig. 5) [51, 52, 54]. Ultrasonography of IgG4related sclerosing cholangitis has revealed wall lesion disappeared. Sarcoidosis-like lesion was shown; e CT shows hilar and mediastinal lymph node swelling and thickening of the bronchial wall and bronchial vascular bundle. f Bronchoscopy shows edema of the bronchial mucosa

thickening of the intra- or extrahepatic bile ducts. Moreover, histological examinations of the bile duct wall in IgG4-related sclerosing cholangitis have shown similar pathology to that observed in pancreatic tissue [55-57]. Inflammation associated with IgG4-related sclerosing cholangitis is found throughout all the layers of the bile duct wall, while inflammation associated with PSC is found predominantly in the inner wall portion, with only slight changes in the outer wall portion of the bile duct. Liver biopsies have shown several IgG4-bearing plasma cell infiltrations in the portal area in IgG4-related sclerosing cholangitis, but only a few in PSC $[20,52,55$ 57].

IgG4-related sclerosing cholangitis sometimes shows slight or no pancreatic lesions, which may lead to a misdiagnosis of PSC [53, 58, 59]. Even without pancreatic swelling, pancreatography sometimes discloses irregular narrowing of the main pancreatic duct (MPD), which suggests that an endoscopic retrograde cholangiopancreatography (ERCP) would be very useful in those situations [59]. 
IgG4-related sclerosing cholangitis with localized bile duct stenosis must be differentiated from bile duct cancer $[60,61]$. Because it is sometimes difficult for cholangiography alone to differentiate between these conditions, it is necessary to perform a careful examination with other tests such as EUS, IDUS, cytology, and tissue biopsy [60-63]. Although a finding of IgG4-bearing plasma cell infiltrations in the bile duct wall supports the diagnosis of IgG4-related

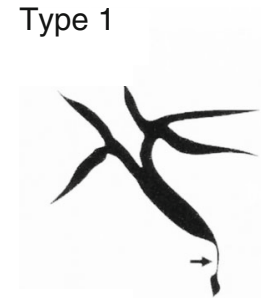

Type 3

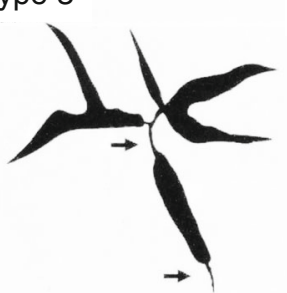

Type 2

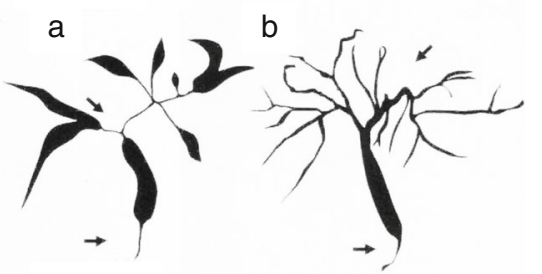

Type 4
Fig. 4 Schematic classification of sclerosing cholangitis associated with AIP, identified with cholangiography. Type 1: stenosis only in the lower part of the common bile duct. Type 2: stenosis in the intrahepatic and extra-hepatic bile ducts; (type 2a) extended narrowing of intrahepatic bile ducts with pre-stenotic dilation; (type $2 b$ ) extended narrowing of intrahepatic bile ducts without pre-stenotic dilation and a reduced number of bile duct branches. Type 3: stenoses both in hilar hepatic lesions and the lower part of the common bile ducts. Type 4: stenosis only in the hilar hepatic lesions (from Ref. [50]) sclerosing cholangitis $[53,58]$, some reports have denied the diagnostic utility of a bile duct biopsy [63]. Characteristic IDUS findings are a thickening of the inner hypoechoic zone and the preservation of the luminal and outer hyperechoic zones [63, 64]. In some studies, IDUS showed a thickening of the bile duct wall, whereas cholangiography showed normal findings [63]. These characteristic findings will aid in differentiating between the two conditions (also see CQII-1.6). IgG4-related sclerosing cholangitis may also exhibit an inflammatory pseudotumor, like an outgrowing tumor of the bile duct [55], which can be misdiagnosed as bile duct cancer.

IgG4-related sclerosing cholangitis is frequently complicated with gallbladder lesions, and thus a thickening of the gallbladder wall can provide a clue for accurate diagnosis [65]. When diagnosing IgG4-related sclerosing cholangitis, it is recommended to refer to the clinical diagnostic criteria for IgG4-related sclerosing cholangitis [66].

\section{CQ-II-1-6. What IDUS findings are characteristic of IgG4-related sclerosing cholangitis?}

- Lower bile duct stenosis associated with AIP is caused by two mechanisms: (1) extrinsic compression by a swollen pancreas head, and (2) a thickening of the bile duct wall. (Level of recommendation: B)

- Upper bile duct changes are observed predominantly in the hilar to intrahepatic bile duct system. In these cases, IDUS shows a thickening of the inner hypoechoic zone. IDUS sometimes reveals wall thickening of the bile duct, whereas cholangiography shows normal findings. (Level of recommendation: B)
Fig. 5 Comparison between cholangiogram characteristics of PSC and sclerosing cholangitis with AIP (from Ref. [54])
PSC

Sclerosing cholangitis with AIP

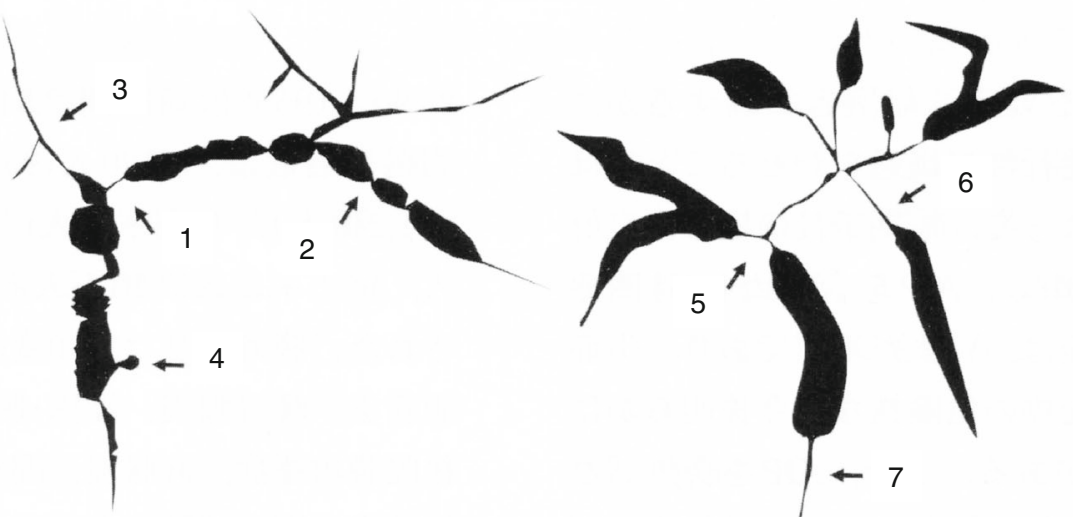

1. band-like stricture

2. beaded appearance

5. segmental stricture

3. pruned-tree appearance

4. diverticulum-like outpouching

6. long stricture with prestenotic dilation

7. stricture of lower CBD 

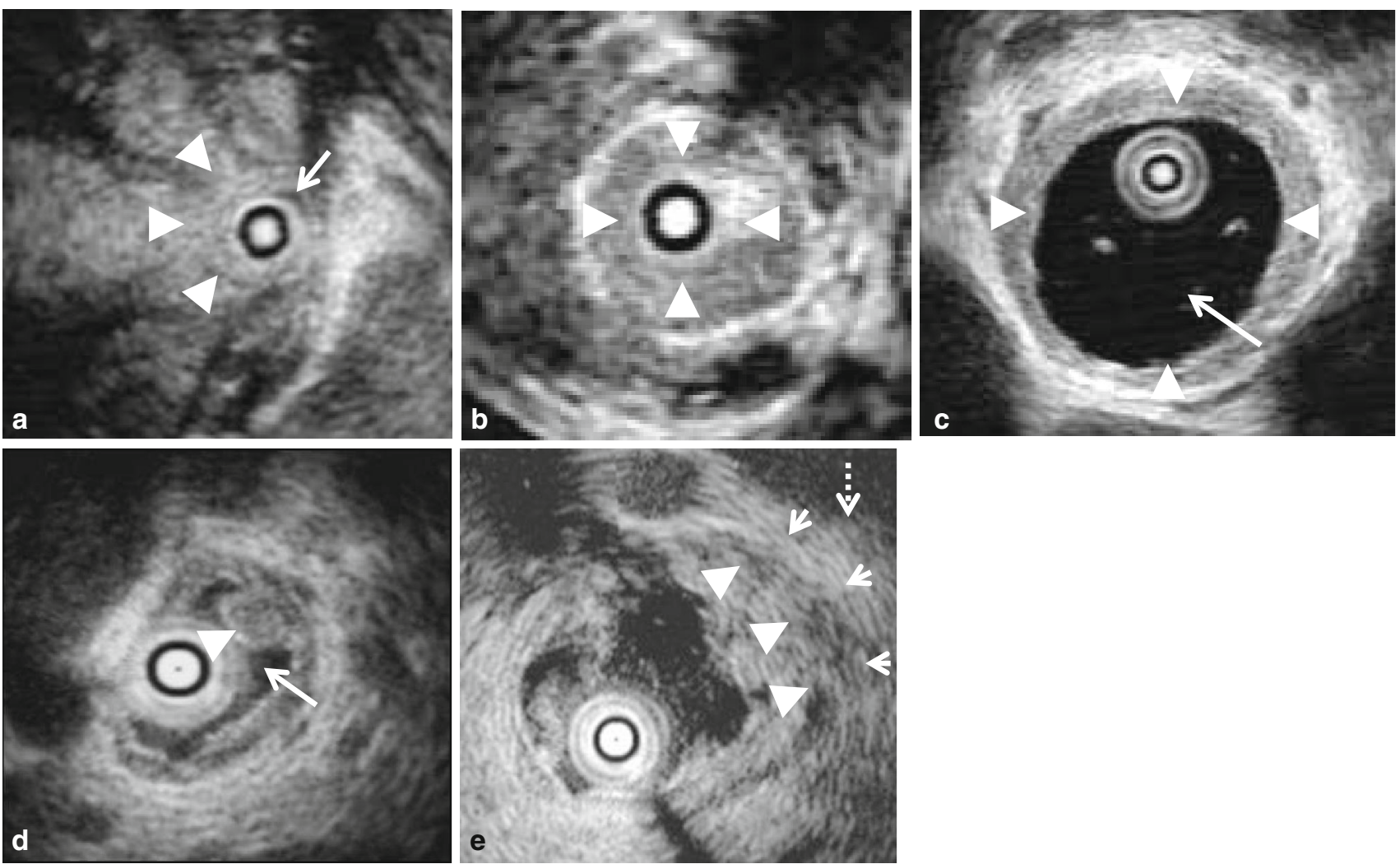

Fig. 6 Intraductal ultrasonography findings. Intraductal ultrasonograph of an intrapancreatic bile duct stenosis found in sclerosing cholangitis associated with AIP: a intraductal ultrasonograph shows lower bile duct stenosis (arrow) caused by extrinsic compression, due to an inflammatory extension from a pancreatic head lesion (arrowheads). b Intraductal ultrasonograph shows lower bile duct stenosis caused by wall thickening of the bile duct (arrowhead). Intraductal ultrasonograph of extrapancreatic bile duct dilation found in sclerosing cholangitis associated with AIP: c intraductal ultrasonograph

Description IgG4-related sclerosing cholangitis is characterized by lower and upper bile duct stenosis. Lower bile duct stenosis is caused by two mechanisms: extrinsic compression from a swollen pancreatic head (Fig. 6a) and thickening of the bile duct wall (Fig. 6b) [28, 63, 67]. Lower bile duct stenosis is frequently observed in cases of pancreatic head swelling, and lower bile duct wall thickening has been reported to be proportional to the degree of bile duct stenosis [67]. In contrast to bile duct cancer, IgG4-related sclerosing cholangitis shows concentric wall thickening and delayed enhancement with Levovist [68, 69].

In IgG4-related sclerosing cholangitis, upper bile duct changes are predominantly observed in the hilar to intrahepatic bile duct system. These changes are reminiscent of those observed in PSC, where IDUS revealed thickening of the inner hypoechoic zone (Fig. 6c) [63]. Although differentiation is difficult with IDUS alone, in PSC, the IDUS shows luminal dilation of upper bile duct (arrow) and homogeneous thickening of inner hypo-echoic zone (arrowheads). d Intraductal ultrasonograph shows upper bile duct stenosis with a slight luminal dilation (arrow), and an irregular surface (arrowhead). Intraductal ultrasonograph of extrapancreatic bile duct stenosis found in extrapancreatic bile duct cancer: e intraductal ultrasonograph shows destruction (arrows) of outer hyper-echoic zone (dotted arrow) due to cancer invasion (arrowheads)

changes include a slight luminal dilation and an irregular surface (Fig. 6d). In contrast to bile duct cancer, for which IDUS showed destruction of outer hyperechoic zone (Fig. 6e), in IgG4-related sclerosing cholangitis, the IDUS commonly shows preservation of the outer hyperechoic zone [63].

In some studies, IDUS showed a thickening of the bile duct wall, whereas cholangiography showed normal findings; the wall of the corresponding region was reported to be thicker than $0.8 \mathrm{~mm}$ [63]. Although bile duct wall thickening is predominantly observed in cancer invasion or PSC [70], biliary drainage also induces thickening of the bile duct wall, and therefore IDUS survey should be performed before biliary drainage [70].

The changes detected by cholangiography in IgG4related sclerosing cholangitis are promptly ameliorated with corticosteroid therapy. The thickening of the bile duct wall detected with IDUS is also ameliorated in parallel 
with decreases in cell infiltration and edema, which result in an increased echo level in a thickened wall. However, unlike the amelioration evident with cholangiography, the changes detected with IDUS tend to persist after corticosteroid therapy.

\section{CQ-II-1-7. What findings are characteristic of retro- peritoneal fibrosis associated with AIP?}

- CT and MRI are commonly used to detect morphologic findings characteristic of retroperitoneal fibrosis. These findings include soft tissue densities that represent masses around the ureter and aorta, near the vertebra, or in the pelvic cavity. (Level of recommendation: B)

- Hydronephrosis and inflammatory aneurysm are sometimes observed as a consequence of retroperitoneal fibrosis. (Level of recommendation: B)

Description AIP-associated retroperitoneal fibrosis is characterized by morphologic findings detected in CT and MRI analyses, including soft tissue densities that represent masses around the aorta (Fig. 2a, b) and the ureter (Fig. 2c), near the vertebra, or in the pelvic cavity. There may also be increased fat density around the superior mesenteric artery [7, 31]. In addition, with positron emission tomography combined with fludeoxyglucose (FDG-PET), intense FDG uptake is typically observed in the corresponding lesions [71]. Histological studies of biopsy specimens have revealed numerous IgG4-bearing plasma cell infiltrations and obstructive phlebitis [7, 72]. Soft tissue masses around the ureter sometimes induce ureteral strictures, which may result in hydronephrosis and irreversible renal failure [73]. These lesions typically respond favorably to corticosteroid treatment [7]. Some cases exhibit periaortitis with adventitial hypertrophy or aneurysm. These findings have been described as an IgG4-related inflammatory abdominal aortic aneurysm [74]. However, it is not certain whether these lesions occurred as a consequence of soft tissue masses around the aorta [22].

\section{CQ-II-1-8. What findings are characteristic of AIP- associated kidney disease?}

- AIP-associated kidney disease is referred to as IgG4related kidney disease; most lesions represent tubulointerstitial nephritis. (Level of recommendation: B).

- Dynamic contrast-enhanced CT and MRI show poorlyenhanced multiple nodules, wedge-shaped lesions, or round lesions in the renal cortex, and mass lesions in the renal pelvis. (Level of recommendation: B).

Description AIP-associated kidney disease is also known as IgG4-related kidney disease. Most lesions are considered tubulointerstitial nephritis [8, 9, 75, 76], and thus a slight urinary finding is common. Renal function is typically normal or slightly impaired, but in some cases, renal failure may occur after severe renal damage. A blood test frequently reveals hypocomplementemia, and it may also show abnormal findings similar to those found in IgG4-related diseases [76]. AIP-associated kidney disease seldom shows glomerular lesions such as membranous nephropathy [77]. Dynamic contrast-enhanced CT or MRI typically reveals poorly enhanced multiple nodules, wedge-shaped lesions, or round lesions in the renal cortex, or a mass in the renal pelvis. A localized renal distribution is characteristic (Fig. 7) [31, 78]. Histological analyses of biopsy specimens typically reveal abundant lymphoplasmacytic and slight eosinophilic infiltrations in the tubulointerstitial region. Renal lesions are relatively localized, and they extend from the deep medulla to the outside of the renal capsule. Some reports have described $\operatorname{IgG}$, IgG4, and complement deposits at the basement membranes of the renal tubule [76, 79]. When diagnosing kidney disease, it is recommended to refer to the diagnostic criteria for IgG4-related kidney disease [80].

\section{II-2. Differential diagnosis}

CQ-II-2-1. What clinical symptoms or findings are useful for differentiating between AIP and pancreatic cancer?

- Useful clinical findings for differentiating between AIP and pancreatic cancer include abdominal pain, weight

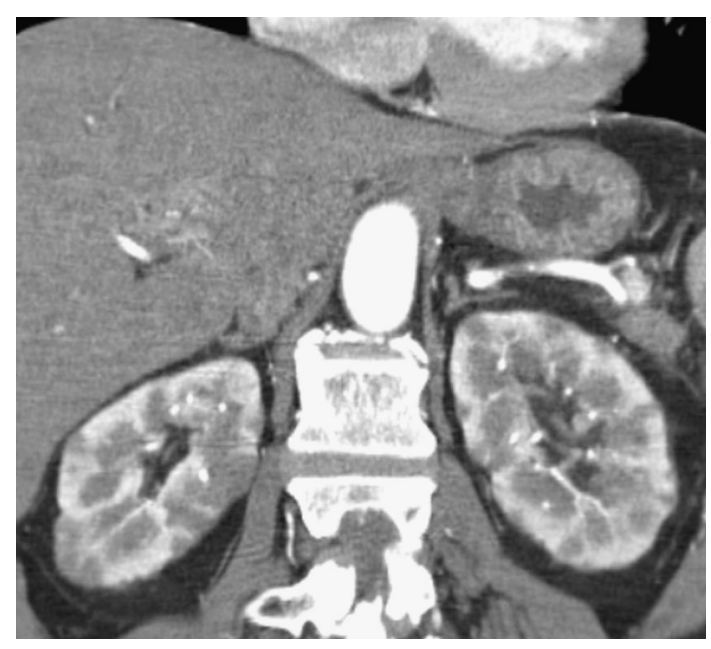

Fig. 7 Kidney disease. Dynamic contrast-enhanced CT (arterial phase) shows poorly-enhanced multiple nodules, wedge-shaped lesions, and round lesions in both renal cortexes 
loss, obstructive jaundice, and extrapancreatic lesions. (Level of recommendation: B)

Description Abdominal pain in pancreatic cancer is severe, persistent, and progressive, sometimes requiring treatment with narcotics. In contrast, abdominal pain in AIP is mild and may be described as simply discomfort of the upper abdomen. [30, 81-87]. Weight loss, which is frequently observed in pancreatic cancer, is rare in AIP, although weight loss in patients with AIP may occur in cases where diabetes mellitus is not under control. Jaundice in pancreatic cancer is progressive; in AIP, jaundice occasionally fluctuates, spontaneously subsides, and responds well to corticosteroid therapy [30, 81-87]. Symptoms associated with various extrapancreatic lesions in AIP include swelling of the lachrymal and salivary glands, jaundice due to sclerosing cholangitis, hydronephrosis due to retroperitoneal fibrosis, hypothyroidism, hypophysitis, and prostatitis [30, 81-87]. In pancreatic cancer, the symptoms associated with apparent extrapancreatic lesions are restricted to lower bile duct stenosis, metastatic lesions, or direct invasions (Table 2) [28].

\section{CQ-II-2-2. Does a high serum IgG4 concentration rule} out the possibility of pancreatic cancer?

- In terms of sensitivity, specificity, and accuracy, elevated IgG4 is the best marker for differentiating between AIP and pancreatic cancer, although a few patients with pancreatic cancer have reported high serum IgG4 concentrations. Therefore, high serum IgG4 concentration cannot completely rule out the presence of pancreatic cancer. (Level of recommendation: B)

Description A high serum IgG4 concentration is commonly found in AIP [53, 84, 87, 88]. In normal subjects, IgG4 comprises 4-6\% of total IgG, and IgG4 serum elevations have been known to occur in a few specific conditions such as allergic diseases, parasite infestations, and pemphigus vulgaris. As serum IgG4 elevations are rarely found in other pancreatic diseases and related autoimmune diseases such as pancreatic cancer, chronic pancreatitis, primary biliary cirrhosis, PSC, and Sjögren's syndrome, a high serum IgG4 concentration is fairly specific to AIP. Furthermore, a finding of numerous IgG4-bearing plasma cell infiltrations in pancreatic tissue is a diagnostic hallmark [7].

In differentiating between AIP and pancreatic cancer, a comparison of various serum markers showed that the best results were obtained with $\mathrm{IgG} 4$, with $86 \%$ sensitivity, $96 \%$ specificity, and $91 \%$ accuracy (Table 3) [28]. IgG4 was adopted as the best marker in Japanese diagnostic
Table 2 Clinical features useful for the differentiation between autoimmune pancreatitis and pancreatic cancer

\begin{tabular}{lll}
\hline & Autoimmune pancreatitis & Pancreatic cancer \\
\hline Abdominal pain & $(-) \sim( \pm)$ & $(+) \sim(+++)$ \\
& Rare & Frequent, progressive \\
Body weight loss & $(-)$ & $(+) \sim(+++)$ \\
Icterus & Frequent, fluctuate & Progressive \\
& PSL responsive & PSL non-responsive \\
Extrapancreatic & Lacrimal gland & Metastatic lesions \\
Lesions & Salivary gland & Surrounding invasion \\
& & tissues \\
& Sclerosing cholangitis & \\
& Retroperitoneal & \\
& fibrosis, etc. & \\
\hline
\end{tabular}

PSL prednisolone

Table 3 Comparison of various markers for differentiating between autoimmune pancreatitis and pancreatic cancer by using identical sera

\begin{tabular}{llll}
\hline & $\begin{array}{l}\text { Sensitivity } \\
(\text { AIP } \\
n=100), \%\end{array}$ & $\begin{array}{l}\text { Specificity } \\
\text { (vs. PC } \\
n=80), \%\end{array}$ & $\begin{array}{l}\text { Accuracy } \\
\text { (vs. PC), }\end{array}$ \\
\hline IgG4 & 86 & 96 & 91 \\
IgG & 69 & 75 & 72 \\
ANA (anti-nuclear & 58 & 79 & 67 \\
antibody) & & & \\
RF (rheumatoid factor) & 23 & 94 & 54 \\
IgG4 + ANA & 95 & 76 & 87 \\
IgG + ANA & 85 & 63 & 75 \\
IgG4 + IgG + ANA & 95 & 63 & 81 \\
IgG4 + RF & 90 & 90 & 90 \\
IgG + RF & 78 & 73 & 76 \\
IgG4 + IgG + RF & 91 & 71 & 82 \\
ANA + RF & 69 & 60 & 78 \\
IgG4 + ANA + RF & 97 & 73 & 86 \\
IgG + ANA + RF & 91 & 61 & 78 \\
IgG4 + IgG + ANA + RF & 97 & 61 & 81 \\
\hline
\end{tabular}

$A I P$ autoimmune pancreatitis, $P C$ pancreatic cancer

criteria for 2006 and 2011 and for the International Consensus Diagnostic Criteria for AIP [83, 89]. However, serum IgG4 elevations or numerous IgG4-bearing plasma cell infiltrations have been found in a few patients with pancreatic cancer [87], suggesting that a high serum IgG4 concentration and increased IgG4-positive plasma cell infiltrations in pancreatic tissue is not completely specific for AIP. Therefore, these findings cannot exclude the presence of pancreatic cancer. 


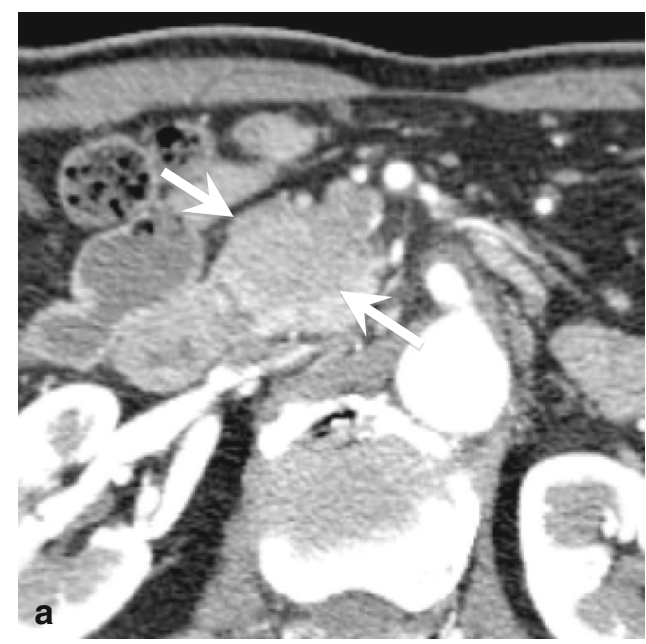

Fig. 8 Localized pancreatic mass in AIP. a Dynamic contrastenhanced CT (arterial phase) shows poorly-enhanced localized mass in the pancreatic head (arrows). b After corticosteroid therapy, the

\section{CQ-II-2-3. What CT, MRI, and FDG-PET findings are useful for differentiating between AIP and pancreatic cancer?}

- Characteristic CT and MRI findings in AIP include a smooth pancreatic margin and a capsule-like rim on the pancreas. (Level of recommendation: A)

- In both in AIP and pancreatic cancer, contrast-enhanced CT often shows delayed enhancement in pancreatic lesions. However, contrast-enhanced images are generally homogeneous in AIP and heterogeneous in pancreatic cancer, and this distinction should aid in the differentiation of the two conditions. (Level of recommendation: B)

- Fat-suppressed T1-weighted MR images of AIP show low signal intensity in pancreatic parenchyma lesions, with speckled/dotted high signal intensity in the lesion. (Level of recommendation: B)

- T2-weighted MR images of AIP sometimes show the main pancreatic duct (MPD) clearly penetrating through a mass lesion. This duct-penetrating sign is absent in pancreatic cancer. (Level of recommendation: A)

- Although localized swelling in AIP is sometimes difficult to distinguish from swellings in pancreatic cancer, the swellings in AIP show marked amelioration after corticosteroid therapy. (Level of recommendation: A)

- FDG-PET reveals intense FDG accumulation at a high rate in the pancreatic lesions of both AIP and pancreatic cancer. In AIP, however, the pancreatic FDG distribution is diffuse with multiple patterns, and FDG is also distributed in extrapancreatic sites, within lachrymal and salivary glands. These findings are useful for differentiating AIP from pancreatic cancer. (Level of recommendation: B)

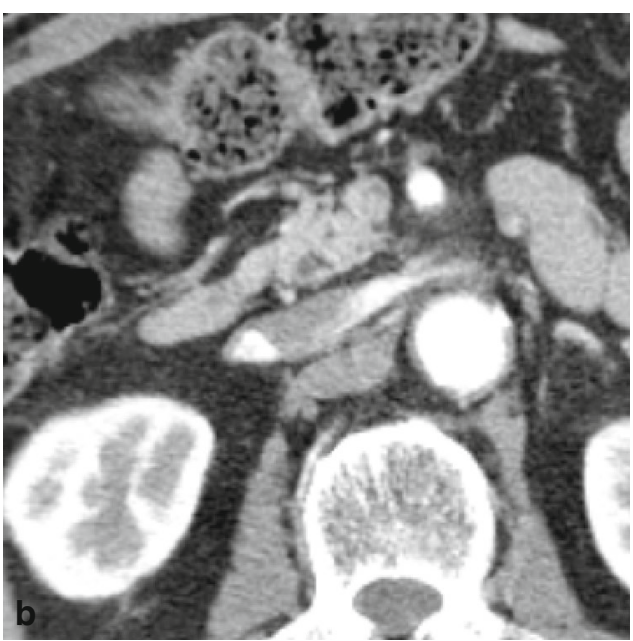

pancreatic swelling decreased in size and the localized mass disappeared

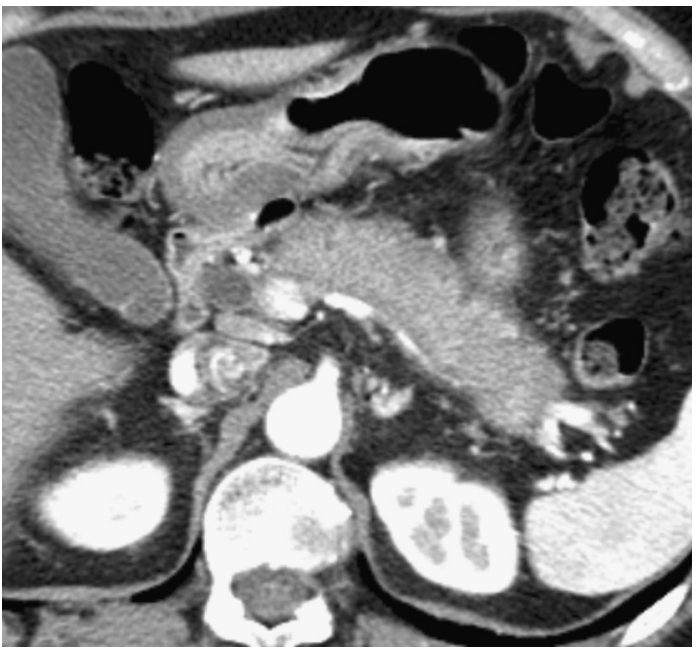

Fig. 9 CT image of typical diffuse type of AIP. Dynamic contrastenhanced CT shows the pancreas with diffuse swelling, a smooth margin, and a capsule-like rim in a patient with AIP

Description AIP sometimes reveals a focal mass on CT and MRI, which should be distinguished from those detected in pancreatic cancer (Fig. 8a). Pancreatic swellings found in AIP improved dramatically after corticosteroid therapy (Fig. 8b). Because pancreatic mass lesions are more common in pancreatic cancer than in AIP, close attention is warranted when diagnosing masses related to AIP requires.

A characteristic finding in AIP is a capsule-like rim that appears on CT and MRI of the pancreatic margin [90-92]. This rim is prominent at the body and tail regions of the pancreas and represents severe fibrotic changes (Fig. 9). $\mathrm{CT}$ and MRI of an aged pancreas have revealed a lobulated margin and a cobblestone-like texture. In contrast, imaging 
of a pancreas with AIP showed a smooth margin, likely due to early stage of the disease (Fig. 9).

Dynamic contrast-enhanced CT with a rapid infusion of contrast material is essential for $\mathrm{CT}$ analysis of pancreatic lesions. In the early (pancreatic parenchymal) phase, the contrast material stains the parenchyma of normal pancreatic tissues; in the late phase, the contrast medium reaches equilibrium between intra- and extravascular fluids. Intense staining in the late phase indicates fibrosis. A contrast-enhanced CT of the AIP pancreas shows delayed homogeneous enhancement in pancreatic mass lesions, which indicates widespread loss of the parenchyma and severe fibrosis (Fig. 10a, b). A contrast-enhanced CT of pancreatic cancer also shows delayed enhancement. However, unlike AIP, the staining pattern shows heterogeneous enhancement (Fig. 10c, d), reflecting necrosis or bleeding in the tumor $[91,93]$.

T1-weighted images are essential for MRI analysis of pancreatic lesions. In combination with the fat-suppressed method, this approach can detect detailed changes in the pancreatic parenchyma. Fat-suppressed T1-weighted MR images of a normal pancreas show high signal intensity compared to those of the liver (Fig. 11a); in contrast, those of a pancreas with AIP show a reduced signal, reflecting the loss of normal parenchyma (Fig. 11b) [28]. Histological analyses of resected AIP-affected pancreatic tissues frequently show a mixture of normal and inflammatory pancreatic tissues [94]. Accordingly, fat-suppressed T1-weighted MR images sometimes show a speckled/dotted hyper-intense region that is enhanced in the pancreatic phase of dynamic contrastenhanced MRI of the affected mass lesion. This finding is useful for distinguishing AIP from pancreatic cancer, as these lesions show high densities in the early phase [95]. T2weighted MR images of the pancreas with AIP generally reveal high-intensity signals, reflecting severe lymphoplasmacytic infiltration. T2-weighted MR images of the pancreas with AIP sometimes show the main pancreatic duct (MPD) clearly penetrating through the mass lesion, which is a useful sign for differentiating between AIP and pancreatic cancer [96] (Fig. 11c, d) [28]. However, reports have indicated that the frequency of this occurrence is no different between a small focal AIP and pancreatic cancer [95].

In AIP, CT and MRI sometimes show wall thickening in the gallbladder and bile duct, even in the absence of duct
Fig. 10 Differences of CT images between localized type of AIP and pancreatic cancer. CT image of localized type of AIP: a dynamic contrastenhanced CT (arterial phase) of AIP-affected pancreas shows a poorly-enhanced, localized mass in the pancreatic tail (arrows) detected in the early phase. $\mathbf{b}$ In the late phase, a delayed, homogeneous enhancement is shown in region of the mass. CT image of pancreatic head cancer: c dynamic contrast-enhanced CT (arterial phase) shows a poorly-enhanced, localized mass in the pancreatic head (arrows). d In the late phase, a delayed, heterogeneous enhancement is detected with a central poorly-enhanced region (arrow) in the mass
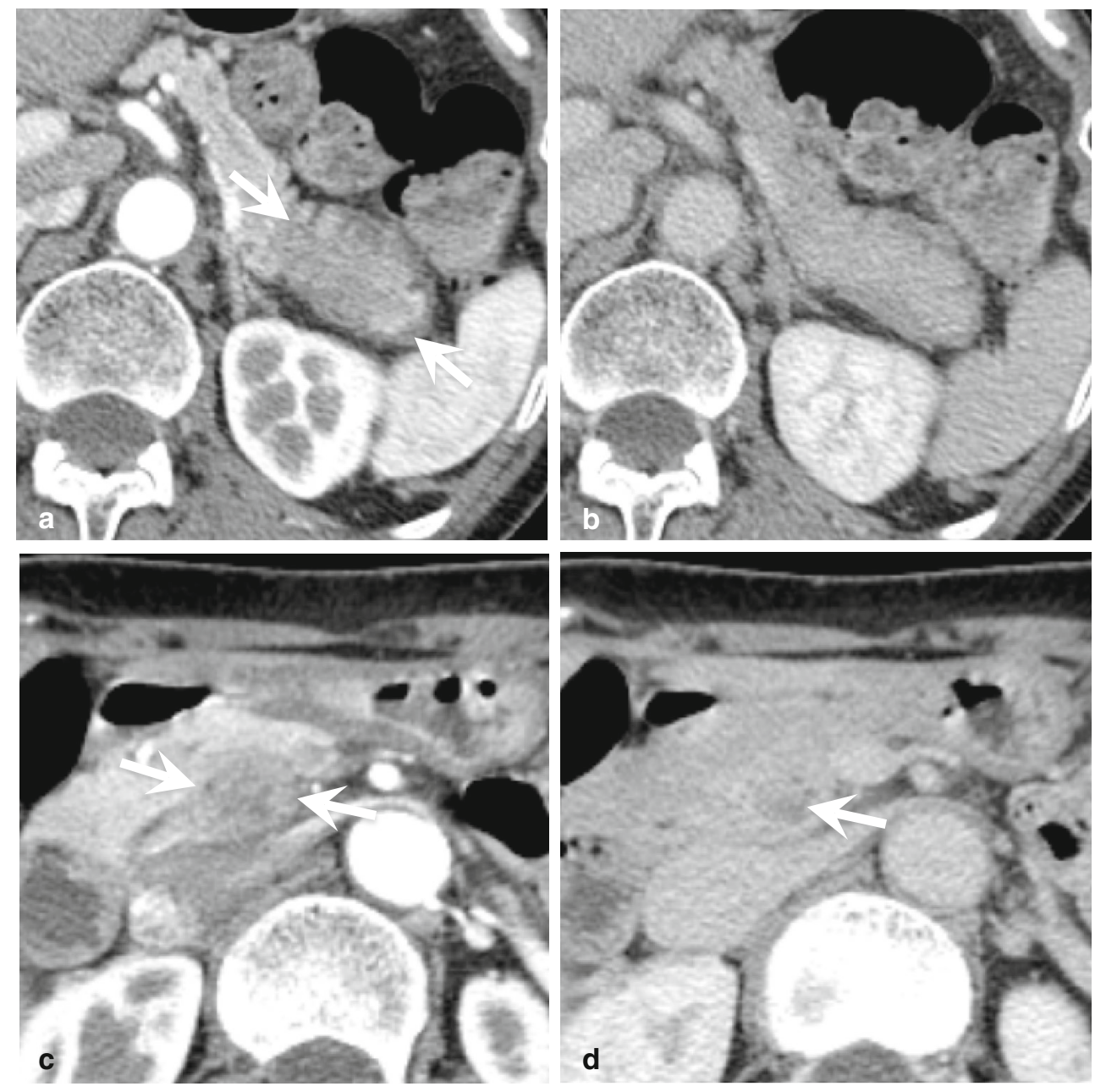
Fig. 11 MRI of the pancreas. a Fat-suppressed T1-weighted MRI of a normal pancreas shows a high signal intensity compared to that of the liver (arrows). b Fat-suppressed T1weighted MRI of an AIPaffected pancreas shows a decreased signal in the swollen pancreatic body and tail (arrows). c Fat-suppressed T1weighted MRI of AIP shows a decreased signal in a pancreatic body mass (arrow). d T2weighted MRI of AIP shows the main pancreatic duct clearly penetrating through the mass (arrow points to the ductpenetrating sign)
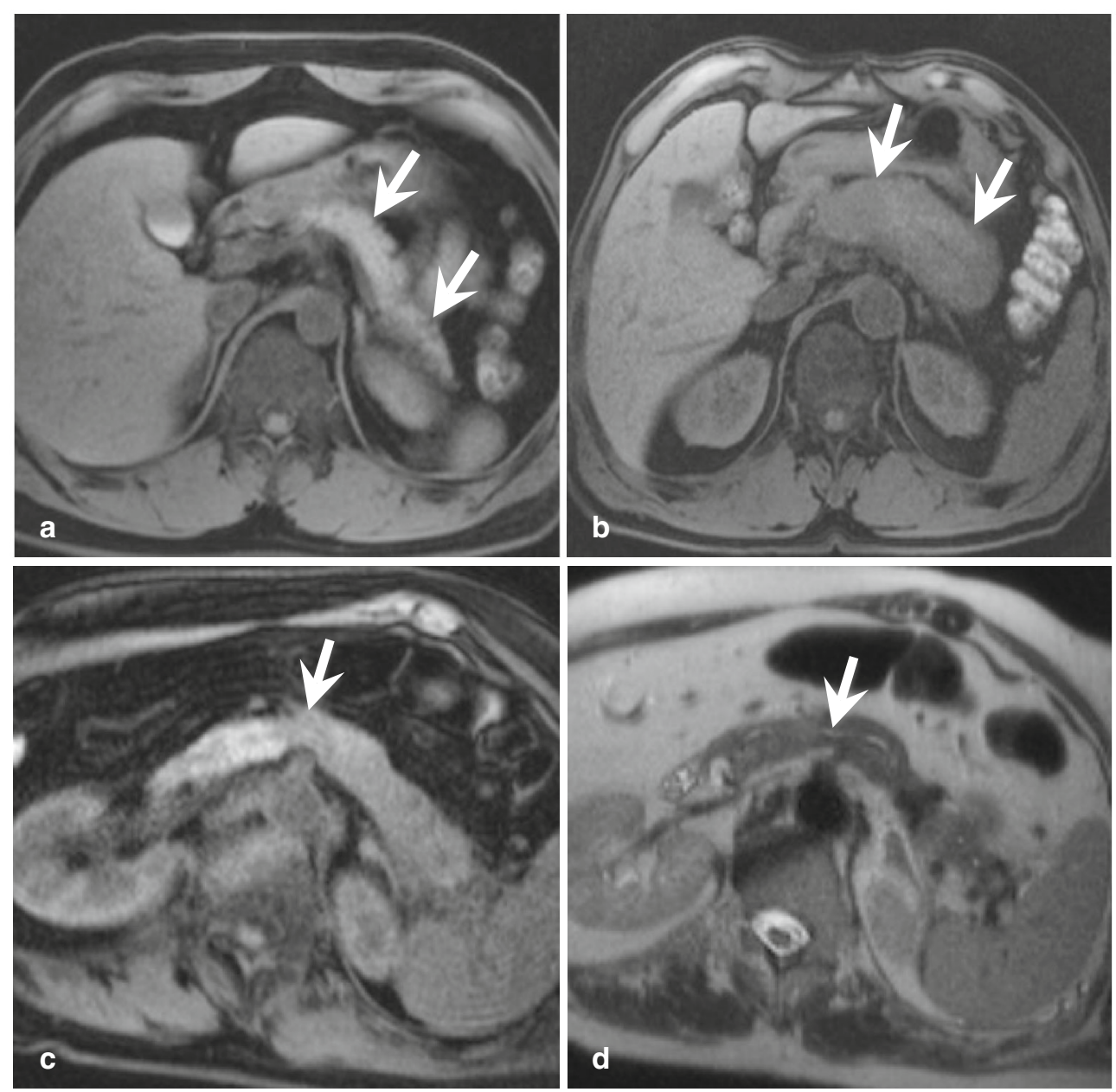

stenosis (Fig. 12) [28, 91, 92]. These findings are rare in pancreatic cancer.

The findings discussed above are characteristic of AIP in an active stage. However, AIP may progress to intraductal stone formation after several relapses, resulting in pancreatic juice stasis and severe calcification. In this instance AIP becomes indistinguishable from ordinary chronic pancreatitis (Fig. 13) [28, 97-99].

Similar to ERCP, magnetic resonance cholangiopancreatography (MRCP) of the pancreas with AIP also shows narrowing of the MPD but with low resolution. ERCP findings that differentiate AIP from pancreatic cancer include a longer stenosis in the MPD (more than $3 \mathrm{~cm}$ ), the presence of branches in the stenosed MPD region, and, after a stenosis of less than $4 \mathrm{~mm}$, a non-dilated MPD [100, 101]. These findings are not possible to discern in cases with MPD obstruction. However, MRCP can image the distal duct even when ERCP shows only obstruction. While MRCP can detect MPD dilation in AIP, it provides comparatively poor images of MPD narrowing or side branches compared to ERCP [102]. Like ERCP, MRCP of the MPD typically shows mild or no distal dilation in AIP (Fig. 14a) [28, 102] but prominent dilation in pancreatic cancer (Fig. 14b).
FDG-PET reveals intense FDG accumulation at a high rate in the pancreatic lesions of both AIP and pancreatic cancer. In AIP, the pancreatic distribution is diffuse, with signals at multiple sites, while the signal is restricted to a solitary site in pancreatic cancer, [32, 103]. FDG accumulation in AIP also appears at extrapancreatic sites, such as the lachrymal and salivary glands or the hilar lymph node. These features are useful for distinguishing between AIP and pancreatic cancer [32, 103, 104]. Another useful differentiating feature in AIP is a rapid decrease in FDG accumulation after corticosteroid therapy [104, 105].

\section{CQ-II-2-4. What EUS findings are useful for differen- tiating between AIP and pancreatic cancer or ordinary chronic pancreatitis?}

- In AIP, a typical EUS of the pancreas shows a relatively diffuse homogeneous hypoechoic pattern and linear or reticular (tortoiseshell pattern) hyperechoic inclusions. (Level of recommendation: B)

- Compared to chronic pancreatitis, EUS in AIP typically shows a homogeneous hypoechoic pattern in the pancreatic parenchyma. EUS rarely shows characteristics 
Fig. 12 CT image of gallbladder and bile duct lesions in AIP. a Dynamic contrastenhanced CT (arterial phase) of AIP-affected tissues shows pancreatic swelling and thickening of the gallbladder wall (arrow). b In the late phase, dynamic contrastenhanced CT shows thickening of the bile duct wall in AIP (arrows)
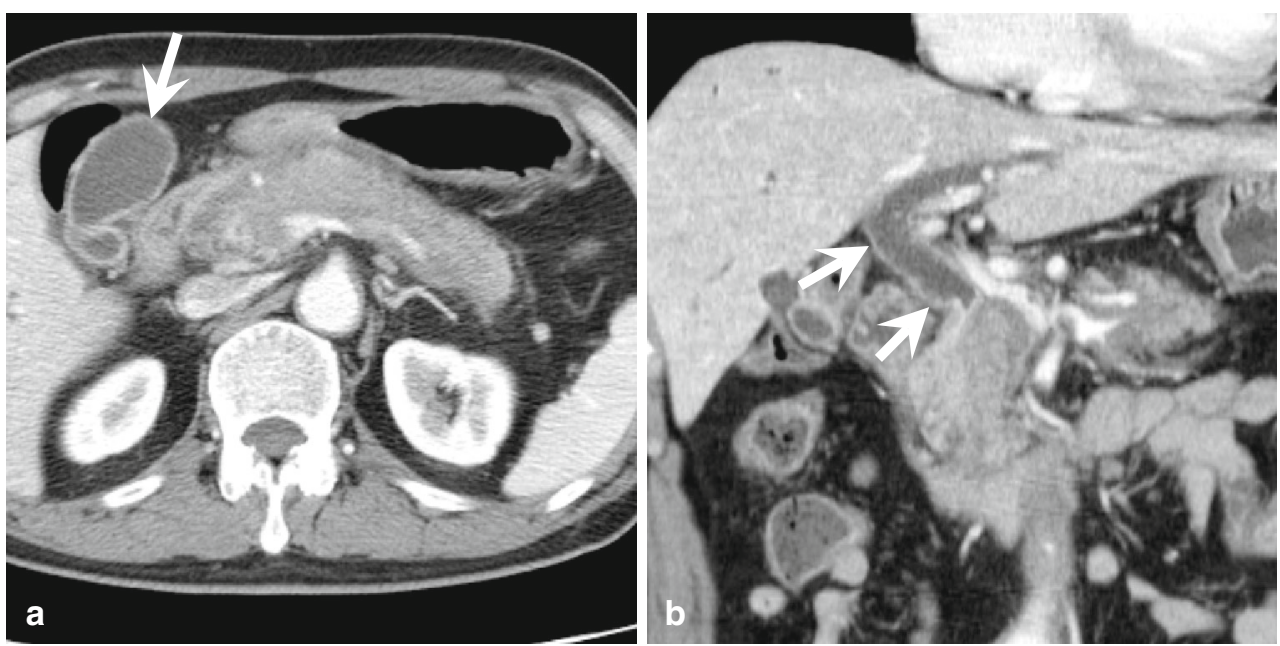

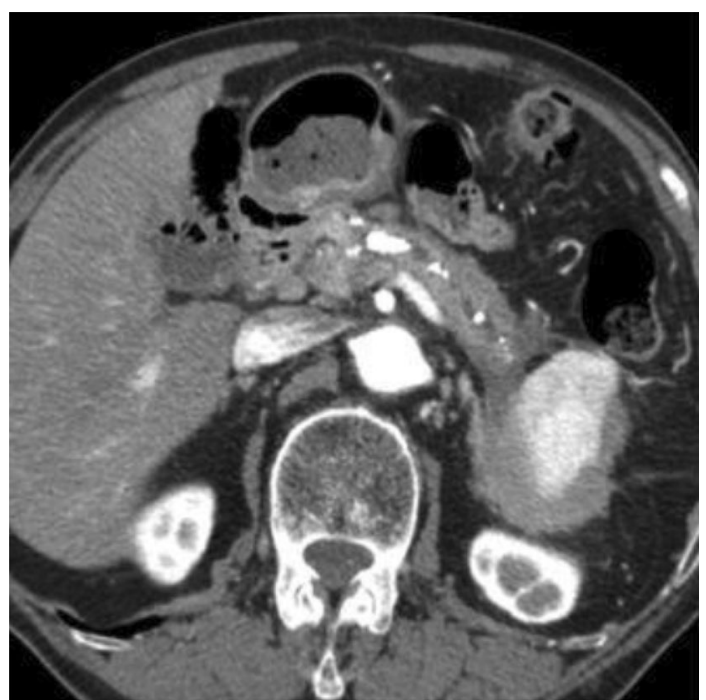

Fig. $13 \mathrm{CT}$ shows an intraductal pancreatic stone in a patient with AIP

like those of chronic pancreatitis (e.g., heterogeneous texture, lobule-shaped margin, calcification, and hyperechoic ductal margin). (Level of recommendation: B)

- EUS of a localized mass in AIP also shows hypoechoic patterns. Linear or reticular (tortoiseshell pattern) hyperechoic inclusions and the duct penetration are useful signs in distinguishing AIP from pancreatic cancer. (Level of recommendation: B)

- EUS with fine-needle aspiration (EUS-FNA) has diagnostic utility for discounting pancreatic cancer. (Level of recommendation: A)

Description Although few studies have described EUS findings that may differentiate between AIP and pancreatic cancer or chronic pancreatitis, some findings that may be useful can be deduced from EUS or US studies of each disease [106-110]. Typical EUS of the pancreas with AIP has revealed a diffuse hypoechoic pattern [106-111] (Fig. 15a) [28], reflecting severe inflammatory cell infiltration. EUS of the pancreas in chronic pancreatitis has shown a heterogeneous echo pattern even when inflammatory changes were severe. Hyperechoic inclusions have been found in both conditions, but in AIP they occurred less frequently and characteristically presented as linear or reticular (tortoiseshell) patterns against the hypoechoic background in the post-acute phase (Fig. 15b) [28]. These findings may represent interlobular fibrosis. Unlike AIP, chronic pancreatitis generally has a lobule-shaped pancreatic margin, a hyperechoic ductal margin, calcification, and cysts. In addition, the hyperechoic inclusions in AIP sometimes promptly disappear with corticosteroid treatment. Although a localized mass with a hypoechoic pattern has been reported in EUS studies of both AIP and pancreatic cancer, only AIP is generally associated with linear or reticular (tortoiseshell pattern) hyperechoic inclusions (Fig. 15c) [28] and the duct-penetrating sign (Fig. 15d) $[28,111]$. While EUS findings of lymph node swelling and vascular invasion are typically associated with pancreatic cancer, it is sometimes difficult to distinguish between the two conditions, and EUS-FNA may be required [112]. EUS-FNA is an excellent diagnostic tool for discounting pancreatic cancer due to its high specificity (98-100\%), but it cannot provide a definitive diagnosis of AIP due to the small sample volume [113-115].

\section{CQ-II-2-5. What pathological findings are useful for differentiating AIP from pancreatic cancer?}

- Histological identification of carcinoma cells is the hallmark for the diagnosis of pancreatic cancer. (Level of recommendation: A)

- Inflammatory reactions can be commonly observed around pancreatic cancer. (Level of recommendation: A) 

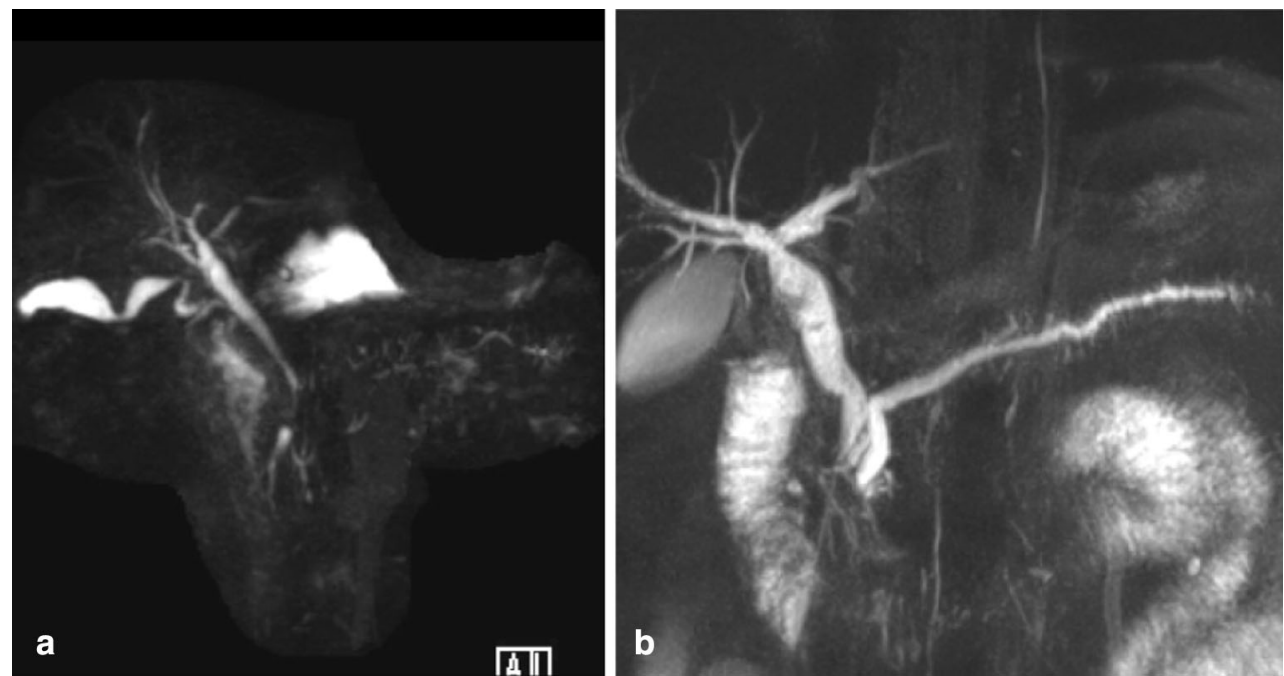

Fig. 14 MRCP image of AIP-affected pancreas and pancreatic cancer. a MRCP shows minor or no distal dilation in AIP-affected MPD after a stenosis. b MRCP shows a prominent dilation in pancreatic cancer-affected MPD after a stenosis
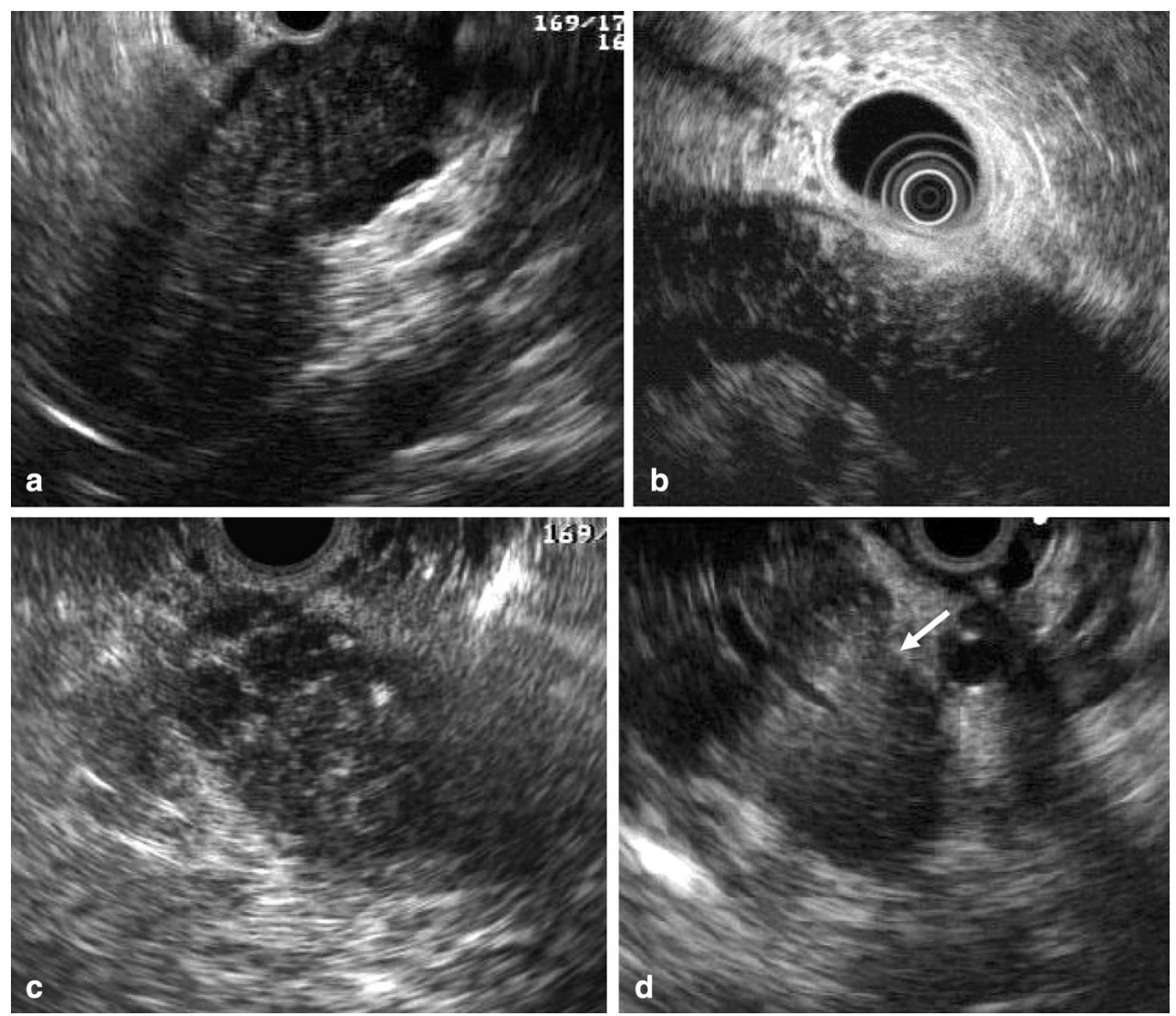

Fig. 15 EUS finding in AIP. a EUS shows a diffuse, hypo-echoic pattern in the swollen pancreas affected by AIP; this finding is rarely observed in cases of chronic pancreatitis. b EUS shows hyper-echoic inclusions, represented by linear or reticular patterns (tortoiseshell pattern) against the hypo-echoic background in the swollen pancreas affected by AIP; these findings are also commonly observed in cases of chronic pancreatitis. c EUS shows a localized mass with a hypoechoic pattern and linear or reticular (tortoiseshell pattern) hyperechoic inclusions in the pancreas of a patient with AIP. d EUS shows the pancreatic duct penetrating through a lesion in the swollen pancreatic parenchyma (arrow points to the duct-penetrating sign) in the pancreas of a patient with AIP 
- Neutrophilic infiltrates, lymphocyte-predominant infiltrates with scarce plasma cells, and proliferation of plump fibroblasts are more common in pancreatic cancer than in AIP, although these findings should not be regarded as sole diagnostic criteria for differentiation. (Level of recommendation: B)

Description Diagnosis of pancreatic cancer by pathological findings may be confirmed by histological identification of carcinoma cells, which is normally easy with resected specimens. Because EUS-FNA is specific for the diagnosis of pancreatic cancer, it provides a useful method to exclude the presence of pancreatic cancer $[113,114]$. However, it is common to observe inflammatory reactions around pancreatic cancer, and cautious interpretation of biopsy specimens with inflammatory changes is necessary to ensure accurate diagnosis of AIP. There is insufficient evidence regarding the differentiation between AIP and pancreatic cancer based on pathological findings. Neutrophilic infiltrates, inflammatory infiltrates and edema in the lobules, proliferation of plump fibroblasts, and lymphocyte-predominant infiltrates with scarce plasma cells are more common in pancreatic cancer than in AIP (Fig. 16a) [28]. Numerous plasma cell infiltration is regarded as a characteristic of AIP, whereas predominant lymphocytic infiltration with scarce plasma cell is preferentially found at inflammatory sites of pancreatic cancer (Fig. 16b) [28]. These findings in isolation should not be regarded as definitive diagnostic indications for AIP. In addition, lymphoid follicles are commonly seen in both pancreatic cancer and AIP, and should not be regarded as a diagnostic hallmark of AIP. While periductal lymphoplasmacytic infiltrates and obstructive phlebitis are characteristic findings for AIP, these are rarely found in biopsy specimens, and histological diagnosis using biopsy specimens is difficult [116]. A few reports showed clear significance of

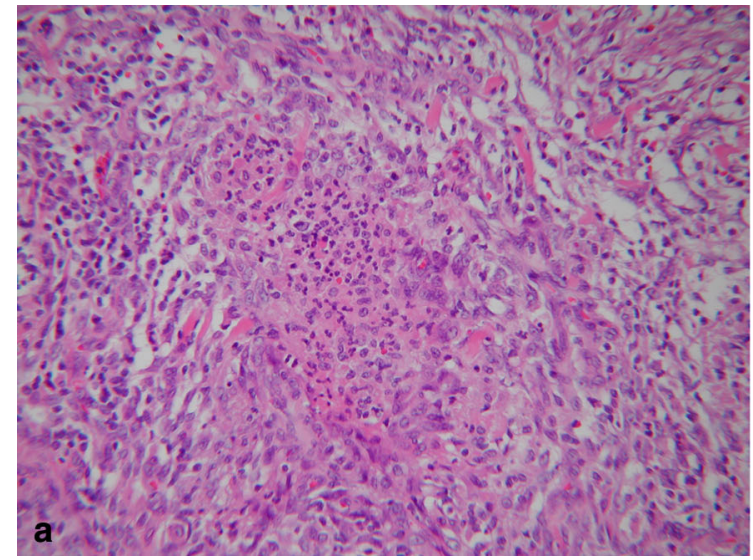

Fig. 16 Histopathological finding in pancreatic cancer. a Tumor biopsy specimen (HE staining) shows a proliferation of plump fibroblasts (desmoplastic reaction). Neutrophilic infiltrates (microabscess) appear in the central area. b Tumor biopsy specimen (HE

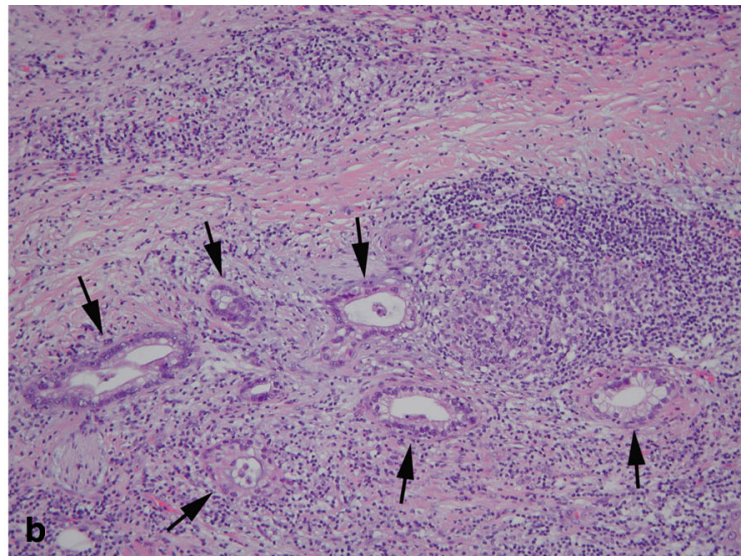

staining) shows predominant lymphocytic infiltration surrounding pancreatic cancer duct cells (arrows). A lymphoid follicle is present at the right

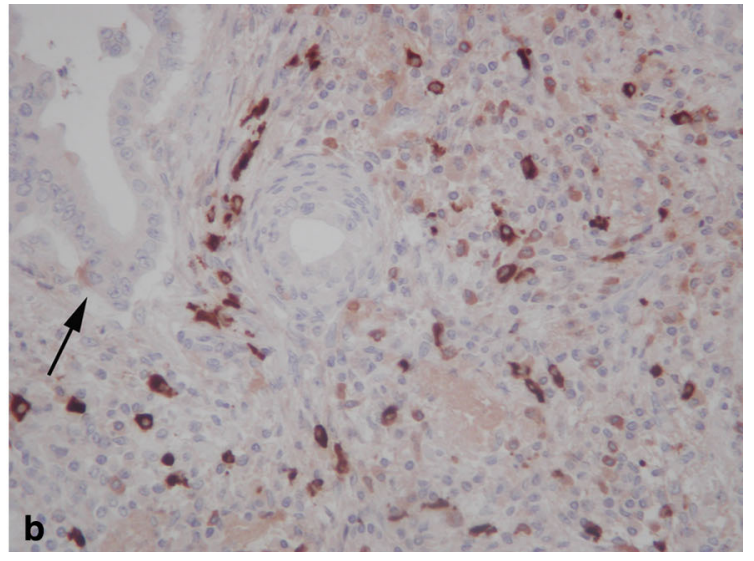

lymphoplasmacytic sclerosing pancreatitis. b Tumor biopsy specimen (IgG4 immunostaining) shows numerous IgG4-positive plasma cells around pancreatic cancer cells (arrow)
Fig. 17 Histopathological finding in pancreatic cancer. a Tumor biopsy specimen (HE staining) shows lymphoplasmacytic infiltration and fibrosis around pancreatic cancer cells; this finding resembles 
EUS-FNA in the histological diagnosis of AIP [115, 117]. Conversely, EUS TruCut biopsy with IgG4 immunostaining was reported to be useful for the diagnosis of AIP [115, 117].

\section{CQ-II-2-6. Are the histological features characteristic of AIP observed in pancreatic cancer?}

- In rare cases, reaction around pancreatic cancer histologically resembles AIP (lymphoplasmacytic sclerosing pancreatitis). (Level of recommendation: B)

- Numerous IgG4-positive plasma cells can be occasionally identified in pancreatic cancer. (Level of recommendation: B)

Description Rare pancreatic cancers reveal histological features that resemble AIP (Fig. 17a) [28, 118-120]. Few histological studies of pancreatic cancer concomitant with AIP have been reported. Irrespective of these characteristic findings, numerous IgG4-positive plasma cells are occasionally identified in pancreatic cancer (Fig. 17b) $[28,116$, 121, 122].

Acknowledgments This study was supported by grant-in-aid for the Intractable Pancreatic Disease from the Ministry of Health, Labour and Welfare of Japan. The authors are grateful to the copyright holders of the artwork in this paper (primarily the Japan Pancreas Society), all of whom kindly provided their consent for reproduction.

Conflict of interest The authors declare that they have no conflict of interest.

\section{Appendix}

The Working Committee of the Japan Pancreas Society (JPS) and the Research Committee for Intractable Pancreatic Disease supported by the Ministry of Health, Labour and Welfare of Japan (RCIPD-MHLWJ):

I. The professional committee for making clinical questions and statements.

Chairperson: Kazuichi Okazaki (Department of Gastroenterology and Hepatology, Kansai Medical University).

Co-Chairpersons: Shigeyuki Kawa (Center for Health, Safety and Environmental Management, Shinshu University), Terumi Kamisawa (Department of Internal Medicine, Tokyo Metropolitan Komagome Hospital).

Committee members:

Tetsuhide Ito (Department of Medicine and Bioregulatory Science, Graduate School of Medical Sciences, Kyushu University), Kazuo Inui (Department of Gastroenterology, Second Teaching Hospital, Fujita Health University), Hiroyuki Irie (Department of Radiology, Faculty of Medicine, Saga University), Takayoshi Nishino
(Department of Gastroenterology, Yachiyo Medical Center, Tokyo Women's Medical University), Kenji Notohara (Department of Anatomic Pathology, Kurashiki Central Hospital), Keishi Kubo (Department of Internal Medicine, Shinshu University School of Medicine), Hirotaka Ohara (Department of Community-Based Medical Education, Nagoya City University Graduate School of Medical Sciences), Atsushi Irisawa (Department of Gastroenterology, Fukushima Medical University Aizu Medical Center), Yasunari Fujinaga (Department of Radiology, Shinshu University School of Medicine), Osamu Hasebe (Department of Gastroenterology, Nagano Municipal Hospital), Isao Nishimori (Nishimori Clinic), Shigeki Tanaka (Department of Acupuncture and Moxibusion, Tokyo Ariake University of Medical and Health Sciences).

II. The expert panelist committee for rating statements by the modified Delphi method

Chairperson: Tooru Shimosegawa

Committee members: Kazuichi Okazaki, Shigeyuki Kawa, Terumi Kamisawa, Tetsuhide Ito, Kazuo Inui, Takayoshi Nishino, Hirotaka Ohara, Isao Nishimori, Shigeki Tanaka

III. The Evaluating Committee

Chairperson: Masao Tanaka (Department of Surgery and Oncology, Kyushu University)

1. Committee members:

Toshimasa Nishiyama (Department of Public Health and Hygiene, Kansai Medical University), Koichi Suda (Department of Pathology, Tokyo-West Tokushukai Hospital), Keiko Shiratori (Department of Gastroenterology, Tokyo Women's Medical University), Kenji Notohara, Keishi Kubo, Hiroshi Yamamoto, Hirotaka Ohara, Atsushi Irisawa, Yasunari Fujinaga, Osamu Hasebe, Shigeki Tanaka.

2. Committee Members of the JPS for Autoimmune Pancreatitis:

Kazushige Uchida (Department of Gastroenterology and Hepatology, Kansai Medical University), Atsushi Kanno (Division of Gastroenterology, Tohoku University Graduate School of Medicine), Kensuke Kubota (Department of Gastroenterology, Yokohama City Unicersity), Shigeru Ko (Department of Systems Medicine, Keio University), Junichi Sakagami (Department of Gastroenterology and Hepatology, Kyoto Prefectural University of Medicine), Kyoko Shimizu (Department of Gastroenterology, Tokyo Women's Medical University), Masanori Sugiyama (Department of Surgery, Kyorin University), Minoru Tada (Department of Gastroenterology, University of Tokyo), Takahiro Nakazawa (Department of Gastroenterology and 
Metabolism, Nagoya City University), Hirokazu Nishino (Department of Gastroenterology and Hepatology, Jikei University School of Medicine), Hideaki Hamano (Medical Informatics Division and Department of Internal Medicine, Gastroenterology, Shinshu University Hospital), Yoshiki Hirooka (Department of Endoscopy, Nagoya University Hospital), Kenji Hirano (Department of Gastroenterology, University of Tokyo), Atsushi Masamune (Division of Gastroenterology, Tohoku University Graduate School of Medicine), Atsuhiro Masuda (Divison of Gastroenterology, Department of Internal Medicine, Kobe University Graduate School of Medicine), Nobumasa Mizuno (Department of Gastroenterology, Aichi Cancer Center Hospital), Koji Yamaguchi (Department of Surgery 1, University of Occupational and Environmental Health), Hitoshi Yoshida (Division of Gastroenterology, Department of Medicine, Showa University School of Medicine).

\section{References}

1. Kamisawa T, Funata N, Hayashi Y, et al. Close relationship between autoimmune pancreatitis and multifocal fibrosclerosis. Gut. 2003;52:683-7.

2. Saegusa H, Momose M, Kawa S, et al. Hilar and pancreatic gallium-67 accumulation is characteristic feature of autoimmune pancreatitis. Pancreas. 2003;27:20-5.

3. Taniguchi T, Ko M, Seko S, et al. Interstitial pneumonia associated with autoimmune pancreatitis. Gut. 2004;53:770 (author reply $770-771$ ).

4. Hirano K, Kawabe T, Komatsu Y, et al. High-rate pulmonary involvement in autoimmune pancreatitis. Intern Med $\mathrm{J}$. 2006;36:58-61.

5. Erkelens GW, Vleggaar FP, Lesterhuis W, et al. Sclerosing pancreato-cholangitis responsive to steroid therapy. Lancet. 1999;354:43-4.

6. Nakazawa $\mathrm{T}$, Ohara $\mathrm{H}$, Yamada $\mathrm{T}$, et al. Atypical primary sclerosing cholangitis cases associated with unusual pancreatitis. Hepatogastroenterology. 2001;48:625-30.

7. Hamano H, Kawa S, Ochi Y, et al. Hydronephrosis associated with retroperitoneal fibrosis and sclerosing pancreatitis. Lancet. 2002;359:1403-4.

8. Takeda S, Haratake J, Kasai T, et al. IgG4-associated idiopathic tubulointerstitial nephritis complicating autoimmune pancreatitis. Nephrol Dial Transpl. 2004;19:474-6.

9. Uchiyama-Tanaka Y, Mori Y, Kimura T, et al. Acute tubulointerstitial nephritis associated with autoimmune-related pancreatitis. Am J Kidney Dis. 2004;43:e18-25.

10. van der Vliet HJ, Perenboom RM. Multiple pseudotumors in IgG4-associated multifocal systemic fibrosis. Ann Intern Med. 2004;141:896-7.

11. Chan SK, Cheuk W, Chan KT, Chan JK. IgG4-related sclerosing pachymeningitis: a previously unrecognized form of central nervous system involvement in IgG4-related sclerosing disease. Am J Surg Pathol. 2009;33:1249-52.

12. Ohara H, Nakazawa T, Sano H, et al. Systemic extrapancreatic lesions associated with autoimmune pancreatitis. Pancreas. 2005;31:232-7.

13. Sugimoto T, Tanaka Y, Morita Y, et al. Is tubulointerstitial nephritis and uveitis syndrome associated with IgG4-related systemic disease? Nephrology (Carlton). 2008;13:89.
14. Komatsu K, Hamano H, Ochi Y, et al. High prevalence of hypothyroidism in patients with autoimmune pancreatitis. Dig Dis Sci. 2005;50:1052-7.

15. Zen Y, Kasahara Y, Horita K, et al. Inflammatory pseudotumor of the breast in a patient with a high serum IgG4 level: histologic similarity to sclerosing pancreatitis. Am J Surg Pathol. 2005;29:275-8.

16. Zen Y, Inoue D, Kitao A, et al. IgG4-related lung and pleural disease: a clinicopathologic study of 21 cases. Am J Surg Pathol. 2009;33:1886-93.

17. Zen Y, Fujii T, Sato Y, et al. Pathological classification of hepatic inflammatory pseudotumor with respect to IgG4-related disease. Mod Pathol. 2007;20:884-94.

18. Shinji A, Sano K, Hamano H, et al. Autoimmune pancreatitis is closely associated with gastric ulcer presenting with abundant IgG4-bearing plasma cell infiltration. Gastrointest Endosc. 2004;59:506-11.

19. Unno H, Saegusa H, Fukushima M, Hamano H. Usefulness of endoscopic observation of the main duodenal papilla in the diagnosis of sclerosing pancreatitis. Gastrointest Endosc. 2002;56:880-4.

20. Umemura T, Zen Y, Hamano H, et al. Immunoglobin G4hepatopathy: association of immunoglobin G4-bearing plasma cells in liver with autoimmune pancreatitis. Hepatology. 2007; 46:463-71.

21. Umemura $\mathrm{T}$, Zen $\mathrm{Y}$, Hamano $\mathrm{H}$, et al. Clinical significance of immunoglobulin G4-associated autoimmune hepatitis. J Gastroenterol. 2011;46 Suppl 1:48-55.

22. Kasashima S, Zen Y, Kawashima A, et al. Inflammatory abdominal aortic aneurysm: close relationship to IgG4-related periaortitis. Am J Surg Pathol. 2008;32:197-204.

23. Yoshimura Y, Takeda S, Ieki Y, et al. IgG4-associated prostatitis complicating autoimmune pancreatitis. Intern Med. 2006;45:897-901.

24. Watanabe T, Fujinaga Y, Kawakami S, et al. Infraorbital nerve swelling associated with autoimmune pancreatitis. Jpn J Radiol. 2011;29:194-201.

25. Nakamura A, Funatomi H, Katagiri A, et al. A case of autoimmune pancreatitis complicated with immune thrombocytopenia during maintenance therapy with prednisolone. Dig Dis Sci. 2003;48:1968-71.

26. Okazaki K, Uchida K, Matsushita M, Takaoka M. How to diagnose autoimmune pancreatitis by the revised Japanese clinical criteria. J Gastroenterol. 2007;42 Suppl 18:32-8.

27. Hamano H, Arakura N, Muraki T, et al. Prevalence and distribution of extrapancreatic lesions complicating autoimmune pancreatitis. J Gastroenterol. 2006;41:1197-205.

28. Kawa S, Okazaki K, Kamisawa T, et al. Japanese consensus guidelines for management of autoimmune pancreatitis: II. Extrapancreatic lesions, differential diagnosis. J Gastroenterol. 2010;45:1264-71.

29. Takuma K, Kamisawa T, Anjiki H, et al. Metachronous extrapancreatic lesions in autoimmune pancreatitis. Intern Med. 2010;49:529-33.

30. Kawa S, Hamano H, Kiyosawa K. Autoimmune pancreatitis and IgG4-related disease. In: Rose N, MacKay I, editors. The autoimmune diseases, 5th edn. St Louis: Academic Press; 2013. pp. 935-949.

31. Fujinaga $\mathrm{Y}$, Kadoya M, Kawa S, et al. Characteristic findings in images of extra-pancreatic lesions associated with autoimmune pancreatitis. Eur J Radiol. 2009;76:228-38.

32. Ozaki Y, Oguchi K, Hamano H, et al. Differentiation of autoimmune pancreatitis from suspected pancreatic cancer by fluorine-18 fluorodeoxyglucose positron emission tomography. J Gastroenterol. 2008;43:144-51.

33. Kamisawa T, Egawa N, Inokuma S, et al. Pancreatic endocrine and exocrine function and salivary gland function in 
autoimmune pancreatitis before and after steroid therapy. Pancreas. 2003;27:235-8.

34. Yamamoto M, Ohara M, Suzuki C, et al. Elevated IgG4 concentrations in serum of patients with Mikulicz's disease. Scand J Rheumatol. 2004;33:432-3.

35. Yamamoto M, Harada S, Ohara M, et al. Clinical and pathological differences between Mikulicz's disease and Sjogren's syndrome. Rheumatology (Oxford). 2005;44:227-34.

36. Kamisawa T, Tu Y, Sasaki R, et al. The relationship of salivary gland function to elevated serum IgG4 in autoimmune pancreatitis. Intern Med. 2007;46:435-9.

37. Manoussakis M, Mouttsopoulos H. Sjoegren syndrome. 4th ed. St Louis: Academic Press; 2006.

38. Kubota K, Wada T, Kato S, et al. Highly active state of autoimmune pancreatitis with Mikulicz disease. Pancreas. 2010;39: e6-10.

39. Masaki Y, Sugai S, Umehara H. IgG4-related diseases including Mikulicz's disease and sclerosing pancreatitis: diagnostic insights. J Rheumatol. 2010;37:1380-5.

40. Inoue D, Zen Y, Abo H, et al. Immunoglobulin G4-related lung disease: CT findings with pathologic correlations. Radiology. 2009;251:260-70.

41. Takato H, Yasui M, Ichikawa Y, et al. Nonspecific interstitial pneumonia with abundant IgG4-positive cells infiltration, which was thought as pulmonary involvement of IgG4-related autoimmune disease. Intern Med. 2008;47:291-4.

42. Hamed G, Tsushima K, Yasuo M, et al. Inflammatory lesions of the lung, submandibular gland, bile duct and prostate in a patient with IgG4-associated multifocal systemic fibrosclerosis. Respirology. 2007;12:455-7.

43. Miyake K, Moriyama M, Aizawa K, et al. Peripheral $\mathrm{CD}^{4+} \mathrm{T}$ cells showing a Th2 phenotype in a patient with Mikulicz's disease associated with lymphadenopathy and pleural effusion. Mod Rheumatol. 2008;18:86-90.

44. Tsushima K, Tanabe T, Yamamoto H, et al. Pulmonary involvement of autoimmune pancreatitis. Eur $\mathrm{J}$ Clin Invest. 2009;39:714-22.

45. Shrestha B, Sekiguchi H, Colby TV, et al. Distinctive pulmonary histopathology with increased IgG4-positive plasma cells in patients with autoimmune pancreatitis: report of 6 and 12 cases with similar histopathology. Am J Surg Pathol. 2009;33:1450-62.

46. Matsui S, Hebisawa A, Sakai F, et al. Immunoglobulin G4related lung disease: clinicoradiological and pathological features. Respirology. 2013;18:480-7.

47. Zen Y, Kitagawa S, Minato H, et al. IgG4-positive plasma cells in inflammatory pseudotumor (plasma cell granuloma) of the lung. Hum Pathol. 2005;36:710-7.

48. Ito M, Yasuo M, Yamamoto $\mathrm{H}$, et al. Central airway stenosis in a patient with autoimmune pancreatitis. Eur Respir J. 2009;33:680-3.

49. Yamamoto H, Yasuo M, Ito M, et al. Clinical features of central airway involvement in autoimmune pancreatitis. Eur Respir J. 2011;38:1233-6.

50. Nakazawa T, Ohara H, Sano H, et al. Schematic classification of sclerosing cholangitis with autoimmune pancreatitis by cholangiography. Pancreas. 2006;32:229.

51. Nakazawa T, Ohara H, Sano $\mathrm{H}$, et al. Clinical differences between primary sclerosing cholangitis and sclerosing cholangitis with autoimmune pancreatitis. Pancreas. 2005;30:20-5.

52. Nishino T, Oyama H, Hashimoto E, et al. Clinicopathological differentiation between sclerosing cholangitis with autoimmune pancreatitis and primary sclerosing cholangitis. J Gastroenterol. 2007;42:550-9.

53. Ghazale A, Chari ST, Zhang L, et al. Immunoglobulin G4associated cholangitis: clinical profile and response to therapy. Gastroenterology. 2008;134:706-15.
54. Nakazawa T, Ohara H, Sano H, Aoki S, Kobayashi S, Okamoto $\mathrm{T}$, et al. Cholangiography can discriminate sclerosing cholangitis with autoimmune pancreatitis from primary sclerosing cholangitis. Gastrointest Endosc. 2004;60:937-44.

55. Zen Y, Harada K, Sasaki M, et al. IgG4-related sclerosing cholangitis with and without hepatic inflammatory pseudotumor, and sclerosing pancreatitis-associated sclerosing cholangitis: do they belong to a spectrum of sclerosing pancreatitis? Am J Surg Pathol. 2004;28:1193-203.

56. Aoki S, Nakazawa $\mathrm{T}$, Ohara $\mathrm{H}$, et al. Immunohistochemical study of autoimmune pancreatitis using anti-IgG4 antibody and patients' sera. Histopathology. 2005;47:147-58.

57. Uehara T, Hamano H, Kawa S, et al. Distinct clinicopathological entity 'autoimmune pancreatitis-associated sclerosing cholangitis'. Pathol Int. 2005;55:405-11.

58. Hamano H, Umemura $\mathrm{T}$, Uehara $\mathrm{T}$, et al. IgG4-related sclerosing cholangitis should be included as an exclusion criterion for the diagnosis of primary sclerosing cholangitis. Am J Gastroenterol. 2007;102:691-2.

59. Hayashi K, Nakazawa T, Ohara H, et al. Autoimmune sclerosing cholangiopancreatitis with little pancreatic involvements by imaging findings. Hepatogastroenterology. 2007;54:2146-51.

60. Tamada K, Kanai N, Wada S. Utility and limitations of intraductal ultrasonography in distinguishing longitudinal cancer extension along the bile duct from inflammatory wall thickening. Abdom Imaging. 2001;26:623-31.

61. Kitajima Y, Ohara H, Nakazawa T. Usefulness of transpapillary bile duct brushing cytology and forceps biopsy for improved diagnosis in patients with biliary strictures. J Gastroenterol Hepatol. 2007;22:1615-20.

62. Arikawa S, Uchida M, Kunou Y, et al. Comparison of sclerosing cholangitis with autoimmune pancreatitis and infiltrative extrahepatic cholangiocarcinoma: multidetector-row computed tomography findings. Jpn J Radiol. 2010;28:205-13.

63. Naitoh I, Nakazawa T, Ohara H, et al. Endoscopic transpapillary intraductal ultrasonography and biopsy in the diagnosis of IgG4related sclerosing cholangitis. J Gastroenterol. 2009;44: 1147-55.

64. Nakazawa T, Ohara H, Ando T, et al. Clinical evaluation of primary sclerosing cholangitis, sclerosing cholangitis with autoimmune pancreatitis and immunoglobulin G4 related sclerosing cholangitis without autoimmune pancreatits. Gut. 2008;57 Suppl 2:A52.

65. Wang WL, Farris AB, Lauwers GY, Deshpande V. Autoimmune pancreatitis-related cholecystitis: a morphologically and immunologically distinctive form of lymphoplasmacytic sclerosing cholecystitis. Histopathology. 2009;54:829-36.

66. Ohara H, Okazaki K, Tsubouchi $\mathrm{H}$, et al. Clinical diagnostic criteria of IgG4-related sclerosing cholangitis 2012. J Hepatobiliary Pancreat Sci. 2012;19:536-42.

67. Hirano K, Tada M, Isayama H, et al. Endoscopic evaluation of factors contributing to intrapancreatic biliary stricture in autoimmune pancreatitis. Gastrointest Endosc. 2010;71:85-90.

68. Hyodo N, Hyodo T. Ultrasonographic evaluation in patients with autoimmune-related pancreatitis. J Gastroenterol. 2003;38:1155-61.

69. Hyodo T, Hyodo N, Yamanaka T. Contrast-enhanced intraductal ultrasonography for thickened bile duct wall. J Gastroenterol. 2001;36:557-9.

70. Tamada K, Tomiyama T, Oohashi A, et al. Bile duct wall thickness measured by intraductal US in patients who have not undergone previous biliary drainage. Gastrointest Endosc. 1999;48:199-203.

71. Otsuka H, Morita N, Yamashita K, Nishitani H. FDG-PET/CT findings of autoimmune pancreatitis associated with idiopathic retroperitoneal fibrosis. Ann Nucl Med. 2007;21:593-6. 
72. Zen Y, Onodera M, Inoue D, et al. Retroperitoneal fibrosis: a clinicopathologic study with respect to immunoglobulin G4. Am J Surg Pathol. 2009;33:1833-9.

73. Kuwatani M, Kawakami H, Makiyama H, et al. Autoimmune pancreatitis with retroperitoneal fibrosis which responded to steroid therapy but was complicated with refractory renal dysfunction. Intern Med. 2007;46:1557-64.

74. Kasashima S, Zen Y, Kawashima A, et al. A new clinicopathological entity of IgG4-related inflammatory abdominal aortic aneurysm. J Vasc Surg. 2009;49:1264-71 (discussion 1271).

75. Saeki T, Saito A, Yamazaki H, et al. Tubulointerstitial nephritis associated with IgG4-related systemic disease. Clin Exp Nephrol. 2007;11:168-73.

76. Saeki T, Nishi S, Imai N, et al. Clinicopathological characteristics of patients with IgG4-related tubulointerstitial nephritis. Kidney Int. 2010;78:1016-23.

77. Saeki T, Imai N, Ito T, et al. Membranous nephropathy associated with IgG4-related systemic disease and without autoimmune pancreatitis. Clin Nephrol. 2009;71:173-8.

78. Takahashi N, Kawashima A, Fletcher JG, Chari ST. Renal involvement in patients with autoimmune pancreatitis: CT and MR imaging findings. Radiology. 2007;242:791-801.

79. Cornell LD, Chicano SL, Deshpande V, et al. Pseudotumors due to IgG4 immune-complex tubulointerstitial nephritis associated with autoimmune pancreatocentric disease. Am J Surg Pathol. 2007;31:1586-97.

80. Kawano M, Saeki T, Nakashima H, et al. Proposal for diagnostic criteria for IgG4-related kidney disease. Clin Exp Nephrol. 2011;15:615-26.

81. Yoshida K, Toki F, Takeuchi T, et al. Chronic pancreatitis caused by an autoimmune abnormality. Proposal of the concept of autoimmune pancreatitis. Dig Dis Sci. 1995;40:1561-8.

82. Okazaki K, Chiba T. Autoimmune related pancreatitis. Gut. 2002;51:1-4.

83. Okazaki K, Kawa S, Kamisawa $\mathrm{T}$, et al. Clinical diagnostic criteria of autoimmune pancreatitis: revised proposal. J Gastroenterol. 2006;41:626-31.

84. Hamano H, Kawa S, Horiuchi A, et al. High serum IgG4 concentrations in patients with sclerosing pancreatitis. New Engl $\mathbf{J}$ Med. 2001;344:732-8.

85. Kawa S, Hamano H, Kiyosawa K. Pancreatitis. In: Rose N, MacKay I, editors. The autoimmune diseases, 4th edn. St Louis: Academic Press; 2006, pp. 779-786.

86. Kawa S, Hamano H. Clinical features of autoimmune pancreatitis. J Gastroenterol. 2007;42 Suppl 18:9-14.

87. Ghazale A, Chari ST, Smyrk TC, et al. Value of serum IgG4 in the diagnosis of autoimmune pancreatitis and in distinguishing it from pancreatic cancer. Am J Gastroenterol. 2007;102:1646-53.

88. Choi EK, Kim MH, Lee TY, et al. The sensitivity and specificity of serum immunoglobulin $\mathrm{G}$ and immunoglobulin $\mathrm{G} 4$ levels in the diagnosis of autoimmune chronic pancreatitis: Korean experience. Pancreas. 2007;35:156-61.

89. Shimosegawa T, Chari ST, Frulloni L, et al. International Consensus Diagnostic Criteria for autoimmune pancreatitis: guidelines of the International Association of Pancreatology. Pancreas. 2011;40:352-8.

90. Irie H, Honda H, Baba S, et al. Autoimmune pancreatitis: $\mathrm{CT}$ and MR characteristics. AJR Am J Roentgenol. 1998;170:1323-7.

91. Takahashi N, Fletcher JG, Fidler JL, et al. Dual-phase CT of autoimmune pancreatitis: a multireader study. AJR Am J Roentgenol. 2008;190:280-6.

92. Kawamoto S, Siegelman SS, Hruban RH, Fishman EK. Lymphoplasmacytic sclerosing pancreatitis (autoimmune pancreatitis): evaluation with multidetector CT. Radiographics. 2008;28:157-70.

93. Chang WI, Kim BJ, Lee JK, et al. The clinical and radiological characteristics of focal mass-forming autoimmune pancreatitis: comparison with chronic pancreatitis and pancreatic cancer. Pancreas. 2009;38:401-8.

94. Sugiyama Y, Fujinaga Y, Kadoya M, et al. Characteristic magnetic resonance features of focal autoimmune pancreatitis useful for differentiation from pancreatic cancer. Jpn J Radiol. 2012;30:296-309.

95. Chandan VS, Iacobuzio-Donahue C, Abraham SC. Patchy distribution of pathologic abnormalities in autoimmune pancreatitis: implications for preoperative diagnosis. Am J Surg Pathol. 2008;32:1762-9.

96. Ichikawa T, Sou H, Araki T. Duct-penetrating sign at MRCP: usefulness for differentiating inflammatory pancreatic mass from pancreatic carcinomas. Radiology. 2001;221:107-16.

97. Takayama M, Hamano H, Ochi Y, et al. Recurrent attacks of autoimmune pancreatitis result in pancreatic stone formation. Am J Gastroenterol. 2004;99:932-7.

98. Maruyama M, Arakura N, Ozaki Y, et al. Risk factors for pancreatic stone formation in autoimmune pancreatitis over a long-term course. J Gastroenterol. 2012;47:553-60.

99. Maruyama M, Arakura N, Ozaki Y, et al. Type 1 autoimmune pancreatitis can transform into chronic pancreatitis: a long-term follow-up study of 73 Japanese patients. Int J Rheumatol. 2013;2013:8.

100. Wakabayashi T, Kawaura Y, Satomura Y, et al. Clinical and imaging features of autoimmune pancreatitis with focal pancreatic swelling or mass formation: comparison with so-called tumor-forming pancreatitis and pancreatic carcinoma. Am J Gastroenterol. 2003;98:2679-87.

101. Nishino T, Oyama H, Toki F, Shiratori K. Differentiation between autoimmune pancreatitis and pancreatic carcinoma based on endoscopic retrograde cholangiopancreatography findings. J Gastroenterol. 2010;45:988-96.

102. Kamisawa T, Tu Y, Egawa N, et al. Can MRCP replace ERCP for the diagnosis of autoimmune pancreatitis? Abdom Imaging. 2009;34:381-4.

103. Lee TY, Kim MH, do Park H, et al. Utility of 18F-FDG PET/CT for differentiation of autoimmune pancreatitis with atypical pancreatic imaging findings from pancreatic cancer. AJR Am J Roentgenol. 2009;193:343-8.

104. Matsubayashi H, Furukawa H, Maeda A, et al. Usefulness of positron emission tomography in the evaluation of distribution and activity of systemic lesions associated with autoimmune pancreatitis. Pancreatology. 2009;9:694-9.

105. Shigekawa M, Yamao K, Sawaki A, et al. Is (18)F-fluorodeoxyglucose positron emission tomography meaningful for estimating the efficacy of corticosteroid therapy in patients with autoimmune pancreatitis? J Hepatobiliary Pancreat Surg. 2009;17:269-74.

106. Farrell JJ, Garber J, Sahani D, Brugge WR. EUS findings in patients with autoimmune pancreatitis. Gastrointest Endosc. 2004;60:927-36.

107. Sahani DV, Kalva SP, Farrell J, et al. Autoimmune pancreatitis: imaging features. Radiology. 2004;233:345-52.

108. Kamisawa T, Egawa N, Nakajima H, et al. Clinical difficulties in the differentiation of autoimmune pancreatitis and pancreatic carcinoma. Am J Gastroenterol. 2003;98:2694-9.

109. Sahai A, Zimmerman M, Aabakken L, et al. Prospective assessment of the ability of endoscopic ultrasound to diagnose, exclude, or establish the severity of chronic pancreatitis found by endoscopic retrograde cholangiopancreatography. Gastrointest Endosc. 1998;48:18-25.

110. Rösch T, Lorenz R, Braig C, Feuerbach S, Siewert J, Schusdziarra V, et al. Endoscopic ultrasound in pancreatic tumor diagnosis. Gastrointest Endosc. 1991;37:347-52.

111. Hoki N, Mizuno N, Sawaki A, et al. Diagnosis of autoimmune pancreatitis using endoscopic ultrasonography. J Gastroenterol. 2009;44:154-9. 
112. Deshpande V, Mino-Kenudson M, Brugge WR, et al. Endoscopic ultrasound guided fine needle aspiration biopsy of autoimmune pancreatitis: diagnostic criteria and pitfalls. Am J Surg Pathol. 2005;29:1464-71.

113. Williams D, Sahai A, Aabakken L, et al. Endoscopic ultrasound guided fine needle aspiration biopsy: a large single centre experience. Gut. 1999;44:720-6.

114. Eloubeidi M, Chen V, Eltoum I. Endoscopic ultrasound-guided fine needle aspiration biopsy of patients with suspected pancreatic cancer: diagnostic accuracy and acute and 30-day complications. Am J Gastroenterol. 2003;98:2663-8.

115. Mizuno N, Bhatia V, Hosoda W, et al. Histological diagnosis of autoimmune pancreatitis using EUS-guided trucut biopsy: a comparison study with EUS-FNA. J Gastroenterol. 2009; 44:742-50.

116. Bang SJ, Kim MH, do Kim H, et al. Is pancreatic core biopsy sufficient to diagnose autoimmune chronic pancreatitis? Pancreas. 2008;36:84-9.
117. Levy MJ, Reddy RP, Wiersema MJ, et al. EUS-guided trucut biopsy in establishing autoimmune pancreatitis as the cause of obstructive jaundice. Gastrointest Endosc. 2005;61:467-72.

118. Inoue H, Miyatani H, Sawada Y, Yoshida Y. A case of pancreas cancer with autoimmune pancreatitis. Pancreas. 2006;33:208-9.

119. Witkiewicz AK, Kennedy EP, Kennyon L, et al. Synchronous autoimmune pancreatitis and infiltrating pancreatic ductal adenocarcinoma: case report and review of the literature. Hum Pathol. 2008;39:1548-51.

120. Motosugi U, Ichikawa T, Yamaguchi H, et al. Small invasive ductal adenocarcinoma of the pancreas associated with lymphoplasmacytic sclerosing pancreatitis. Pathol Int. 2009;59:744-7.

121. Deshpande V, Chicano S, Finkelberg D, et al. Autoimmune pancreatitis: a systemic immune complex mediated disease. Am J Surg Pathol. 2006;30:1537-45.

122. Zhang L, Notohara K, Levy MJ, et al. IgG4-positive plasma cell infiltration in the diagnosis of autoimmune pancreatitis. Mod Pathol. 2007;20:23-8. 